International Journal of Pure and Applied Mathematics

Volume 93 No. $6 \quad 2014,929-979$

ISSN: 1311-8080 (printed version); ISSN: 1314-3395 (on-line version)

url: http://www.ijpam.eu

doi: http://dx.doi.org/10.12732/ijpam.v93i6.14

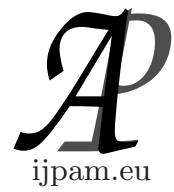

\title{
REPRESENTATION THEOREMS IN A 4-DIMENSIONAL EUCLIDEAN SPACE. \\ THE CASE WITH ONLY SKEW-SYMMETRIC TENSORS
}

\author{
M.C. Carrisi ${ }^{1}$, S. Montisci ${ }^{2}$, S. Pennisi ${ }^{3} \S$ \\ Dipartimento di Matematica ed Informatica \\ Università di Cagliari \\ Via Ospedale 72, 09124, Cagliari, ITALY
}

\begin{abstract}
In a 4-dimensional Euclidean space, representation theorems have been recently obtained for isotropic functions depending on an arbitrary number of scalars, skew-symmetric second order tensors and symmetric second order tensors; the cases has been treated where at least one of these last ones has an eigenvalue with multiplicity 1 or two distinct eigenvalues with multiplicity 2 . The case with at least a non null vector, among the independent variables, was already treated in literature. There remain the case where every symmetric tensor has an eigenvalue with multiplicity 4; but, in this case, it plays a role only through its trace. Consequently, it remains the case where the independent variables, besides scalars, are skew-symmetric tensors. This case is treated in the present paper. As in the other cases, the result is a finite set of scalar valued isotropic functions such that every other scalar function of the same variables can be expressed as a function of the elements of this set. Similarly, a set of tensor valued isotropic functions is found such that every other tensor valued function of the same variables can be expressed as a linear combination, trough scalar coefficients, of the elements of this set. This result is achieved both for symmetric functions, and for skew-symmetric functions.
\end{abstract}

\section{AMS Subject Classification: 15A72}

Key Words: representations theorems, scalar valued isotropic functions, skew-symmetric second order tensors, symmetric second order tensors

\footnotetext{
Received: June 1, 2014

${ }^{\S}$ Correspondence author
}

(C) 2014 Academic Publications, Ltd. url: www.acadpubl.eu 


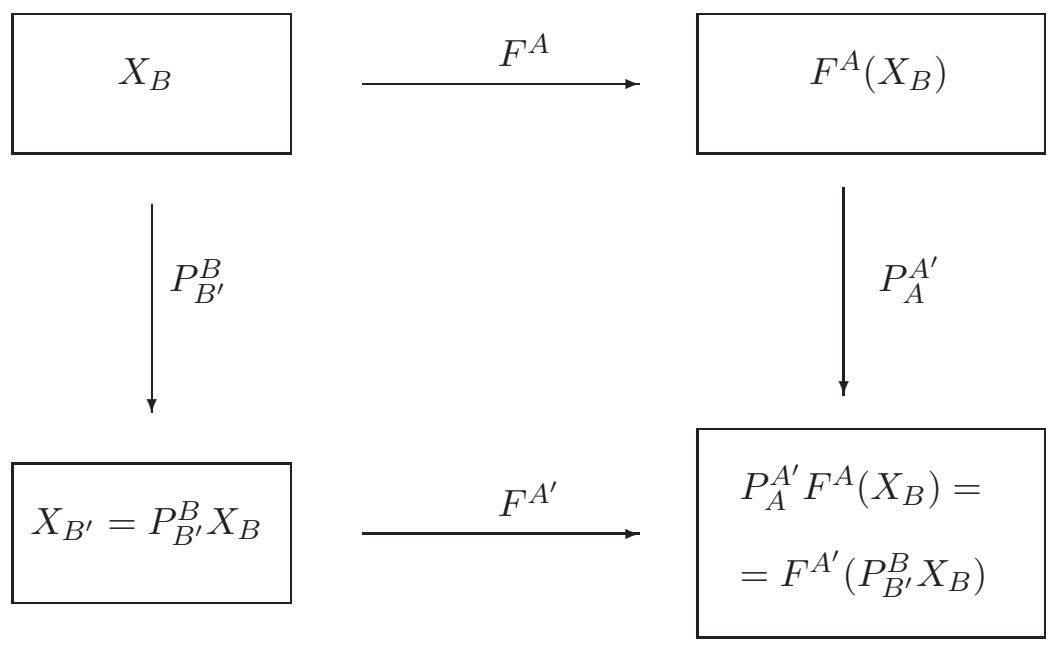

Figure 1: The condition defining isotropic functions.

\section{Introduction}

Representation theorems are the mathematical tool by which to impose the physical principle requiring that the laws of physics do not depend on the observer. Now these laws are expressed in terms of tensors $F^{\alpha_{1} \cdots \alpha_{n}}$ which, in turns, are functions of other tensors $X_{\beta_{1} \cdots \beta_{m}}$; moreover, we know the transformation laws of the components of a tensor, when the basis of the vectorial space is changed. Therefore, the above mentioned requirement amounts in imposing that the diagram in Figure 1 is commutative, where $P_{\alpha}^{\alpha^{\prime}}$ is the orthonormal matrix of the change of basis and the multindex notation has been used, so that $X_{B}$ denotes $X_{\beta_{1} \cdots \beta_{m}}, F^{A}$ denotes $F^{\alpha_{1} \cdots \alpha_{n}}, P_{A}^{A^{\prime}}$ denotes $P_{\alpha_{1}}^{\alpha_{1}^{\prime}} \cdots P_{\alpha_{n}}^{\alpha_{n}^{\prime}}$ and similar. In other words, proceeding from the upper left corner of the figure and moving to the right hand side, we start from the independent variables $X_{\beta_{1} \cdots \beta_{m}}$ in the reference frame $\Sigma$, then we apply to them the function $F^{\alpha_{1} \cdots \alpha_{n}}$, in $\Sigma$; after that, proceeding to the lower side, we transform the result in the reference $\Sigma^{\prime}$. Following the other side of the diagram, we transform the independent variables in $\Sigma^{\prime}$; on the transformed variables, we apply the function $F^{\alpha_{1}^{\prime} \cdots \alpha_{n}^{\prime}}$, in 
$\Sigma^{\prime}$. We require that the result is the same.

What has already been done, about this, in literature? Regarding the framework of a 3-dimensional Euclidean vectorial space, in [1], [2] the case has been considered with $n=0$ (an arbitrary number of scalars), $n=1$ (an arbitrary number of vectors), $n=2$ (an arbitrary number of second order tensors, some of which are symmetric and the remaining ones are skew-symmetric); similar values have been considered for $m$, that is to distinguish the different types of tensorial functions. The result is a set $S^{0}$ of particular scalar functions such that every other scalar function of the same variables can be expressed as a function of the elements of $S^{0}$; similarly, for the other values of $m$ we obtain a set $S^{m}$ of particular tensorial functions of order $m$ such that every other tensorial function of the same order, and depending on the same variables, can be expressed as a linear combination of the elements of $S^{m}$ through scalar coefficients. The sets $S^{m}$ are called "representations"; they are called "irreducible" if no proper subset satisfies the same property. In [3] Boheler proved that the representations exposed in [1] are not irreducible, showing also some their redundant elements. In [4] Pennisi and Trovato proved that once eliminated the elements indicated by Boheler, the remaining elements furnish a complete and irreducible representation. In [5] complete representations were found also for the case $m=3$, that is third order tensorial functions.

Regarding the case of a 4-dimensional vectorial space, Pennisi and Trovato furnished in [6] complete representations, but only for the case of a pseudoEuclidean vectorial space and with the hypothesis that, among the independent variables, there is a time-like 4-vector. Obviously, their result holds also in the case of a 4-dimensional Euclidean vectorial space when, among the independent variables, there is a 4 -vector different from zero. In references [7], [8], [9] the case has been considered where there are no vectors among the independent variables, but among them, there is at least a symmetric second order tensor $\mathcal{A}$ endowed with an eigenvalue $a$ with multiplicity 1 . In [10] the new case has been considered where no symmetric tensor has eigenvalues with multiplicity 1 , but there is at least one symmetric tensor with two distinct eigenvalues.

There remains to consider only the case where every symmetric tensor $\mathcal{A}$ has a eigenvalue with multiplicity four; in this case we have $\mathcal{A}=\mathcal{I}(\operatorname{tr} \mathcal{A})$ so that $\mathcal{A}$ plays a role only through the scalar $\operatorname{tr} \mathcal{A}$. In other words, it remains to consider only the case where all the independent variables, besides scalars, are skew-symmetric tensors. This is the subject of the present work.

Some properties of second order skew-symmetric tensors will be needed; so they will described in section 2, while their proofs is shifted in the Appendix. The treatment of the principal part of this subject is splitted in three cases 
and they are treated in sections 3,4 and 5; each of them is furtherly splitted in subcases and the following notation will be used: "Subcase (a,b) denotes that we are considering the subcase b of the case (a); similarly, "subcase (a,b,c) denotes that we are considering the subcase c of the subcase $(a, b)$.

We report now in table $S$ the result of this work for the set $S^{0}$; the result for the set $S^{2}$ is reported in table $S y$ for symmetric functions, and in table $S k$ for skew-symmetric functions.

There is not a set $S^{1}$ because all the vectorial functions are zero when there is no vector among the independent variables.

\section{The set $S$ : Representations for scalar functions.}

- For every tensor: $\operatorname{tr} \mathcal{V}^{2}, t r \mathcal{V}^{4}$,

- For every couple of tensors: $\operatorname{tr} \mathcal{V} \mathcal{W}, \operatorname{tr} \mathcal{V}^{3} \mathcal{W}, \operatorname{tr} \mathcal{V}^{2} \mathcal{W}^{2}, \operatorname{tr} \mathcal{V} \mathcal{W} \mathcal{W}, \operatorname{tr} \mathcal{V}^{2} \mathcal{W}^{2}$ $\mathcal{V}^{2}, \operatorname{tr} \mathcal{V}^{2} \mathcal{W}^{2} \mathcal{V}^{2} \mathcal{W}^{2}$

- For every set of three tensors: $\operatorname{tr}\left(\mathcal{W}^{2} \mathcal{V}^{2} \mathcal{W U}+\mathcal{V}^{2} \mathcal{W}^{3} \mathcal{U}\right), \operatorname{tr}\left(\mathcal{V}^{2} \mathcal{W}^{2} \mathcal{V}^{2} \mathcal{W U}\right)$ , $\operatorname{tr} \mathcal{W}(\mathcal{V U}-\mathcal{U V})$ $\operatorname{tr} \mathcal{W}^{3}(\mathcal{V U}-\mathcal{U V}), \operatorname{tr} \mathcal{V}^{2} \mathcal{W}^{2} \mathcal{V}^{2} \mathcal{W}(\mathcal{V U}-\mathcal{U} \mathcal{V})$ $\operatorname{tr}\left(\mathcal{W}^{2} \mathcal{V}^{2} \mathcal{W}+\mathcal{V}^{2} \mathcal{W}^{3}\right)(\mathcal{V U}-\mathcal{U V}), \operatorname{tr} \mathcal{W} \mathcal{U} \mathcal{V}$ $\operatorname{tr} \mathcal{W} \mathcal{V}(\mathcal{U}-\mathcal{U V}) \mathcal{V}, \operatorname{tr} \mathcal{V}^{2}(\mathcal{W U}+\mathcal{U} \mathcal{W}) \mathcal{V}^{2}(\mathcal{W U}+\mathcal{U} \mathcal{W})$ $\operatorname{tr} \mathcal{V}^{2}(\mathcal{W U}+\mathcal{U} \mathcal{W})^{2}, \operatorname{tr} \mathcal{V}^{2}(\mathcal{W U}+\mathcal{U} \mathcal{W})$ $\operatorname{tr} \mathcal{V}^{2} \mathcal{W}(\mathcal{V U}-\mathcal{U V}), \operatorname{tr} \mathcal{V}^{2} \mathcal{W}^{2} \mathcal{U}, \operatorname{tr} \mathcal{V}^{2} \mathcal{W}^{2} \mathcal{V}^{2} \mathcal{U}^{2}, \operatorname{tr} \mathcal{V} \mathcal{W}^{2} \mathcal{V}^{2} \mathcal{U}^{2}$

- For every set of four tensors: $\operatorname{tr} \mathcal{U} \mathcal{V W Z}, \operatorname{tr} \mathcal{V}^{2} \mathcal{W} \mathcal{Z}, \operatorname{tr} \mathcal{V}^{2} \mathcal{W}^{2} \mathcal{V}^{2} \mathcal{Z}$, $\operatorname{tr}(\mathcal{V W}+\mathcal{W} \mathcal{V})(\mathcal{Z} \mathcal{T}+\mathcal{T} \mathcal{Z})(\mathcal{V} \mathcal{W}+\mathcal{W} \mathcal{V})(\mathcal{Z} \mathcal{T}+\mathcal{T} \mathcal{Z}), \operatorname{tr}(\mathcal{V} \mathcal{W}+\mathcal{W} \mathcal{V})(\mathcal{Z} \mathcal{T} \mathcal{Z} T$ $\mathcal{T} \mathcal{Z} \mathcal{T})$

besides all the possible permutation of $\mathcal{V}, \mathcal{W}, \mathcal{Z}, \mathcal{T}$ except those which give redundant terms.

\section{The set $S y$ : Representation for symmetric second order tensorial functions.}

- I ,

- For every tensor: $\mathcal{V}^{2}$,

- For every couple of tensors: $\mathcal{W} \mathcal{V}+\mathcal{V} \mathcal{W}, \mathcal{V}^{2} \mathcal{W}+\mathcal{V} \mathcal{W} \mathcal{V}^{2}, \mathcal{V}^{2} \mathcal{W}-\mathcal{W} \mathcal{V}^{2}$ $\mathcal{V}^{2} \mathcal{W}^{2}+\mathcal{W}^{2} \mathcal{V}^{2}$ $\mathcal{V}^{2} \mathcal{W}^{2} \mathcal{V}^{2} \mathcal{W}-\mathcal{W} \mathcal{V}^{2} \mathcal{W}^{2} \mathcal{V}^{2}, \mathcal{W}^{2} \mathcal{V}^{2} \mathcal{W}-\mathcal{W} \mathcal{V}^{2} \mathcal{W}^{2}+\mathcal{V}^{2} \mathcal{W}^{3}-\mathcal{W}^{3} \mathcal{V}^{2}, \mathcal{V}^{2} \mathcal{W}^{2} \mathcal{V}^{3}-$ $\mathcal{V}^{3} \mathcal{W}^{2} \mathcal{V}^{2}$ 
$\mathcal{V}^{2} \mathcal{W}^{2} \mathcal{V}^{2}, \mathcal{V}^{3} \mathcal{W} \mathcal{V}-\mathcal{V} \mathcal{W} \mathcal{V}^{3}, \mathcal{W} \mathcal{V} \mathcal{W}^{2} \mathcal{V}^{2} \mathcal{W}-\mathcal{W} \mathcal{V}^{2} \mathcal{W}^{2} \mathcal{V} \mathcal{W}, \mathcal{W} \mathcal{V} \mathcal{W}^{2} \mathcal{V}^{2}+$ $\mathcal{V}^{2} \mathcal{W}^{2} \mathcal{V W}$ $\mathcal{V}^{2} \mathcal{W}^{2} \mathcal{V}-\mathcal{V} \mathcal{W}^{2} \mathcal{V}^{2}$

- For every set of three tensors:

$\mathcal{V}^{2}(\mathcal{W Z}+\mathcal{Z} \mathcal{W}) \mathcal{V}^{3}-\mathcal{V}^{3}(\mathcal{W Z}+\mathcal{Z} \mathcal{W}) \mathcal{V}^{2}$ $\mathcal{V}^{2}(\mathcal{W Z}+\mathcal{Z} \mathcal{W}) \mathcal{V}+(\mathcal{W} \mathcal{Z}+\mathcal{Z} \mathcal{W}) \mathcal{V}^{3}-\mathcal{V}^{3}(\mathcal{W} \mathcal{Z}+\mathcal{Z} \mathcal{W})-\mathcal{V}(\mathcal{W} \mathcal{Z}+\mathcal{Z} \mathcal{W}) \mathcal{V}^{2}$

$(\mathcal{W Z}+\mathcal{Z} \mathcal{W}) \mathcal{V}-\mathcal{V}(\mathcal{W Z}+\mathcal{Z} \mathcal{W}), \mathcal{V}^{2}(\mathcal{W} \mathcal{Z}+\mathcal{Z} \mathcal{W}) \mathcal{V}^{2}, \mathcal{V}^{2}(\mathcal{W} \mathcal{Z}+\mathcal{Z} \mathcal{W})+$ $(\mathcal{W Z}+\mathcal{Z} \mathcal{W}) \mathcal{V}^{2}$

- For every set of four tensors: $\mathcal{V} \mathcal{W}(\mathcal{Z} \mathcal{T}+\mathcal{T} \mathcal{Z}) \mathcal{W} \mathcal{V}$,

$\mathcal{V W}(\mathcal{Z} \mathcal{T}+\mathcal{T} \mathcal{Z})+(\mathcal{Z} \mathcal{T}+\mathcal{T} \mathcal{Z}) \mathcal{W} \mathcal{V}$

$\mathcal{V W}(\mathcal{Z} \mathcal{T}+\mathcal{T} \mathcal{Z}) \mathcal{W}-\mathcal{W}(\mathcal{Z} \mathcal{T}+\mathcal{T} \mathcal{Z}) \mathcal{W} \mathcal{V}, \mathcal{W} \mathcal{Z} \mathcal{W}+\mathcal{W} \mathcal{Z} \mathcal{W}$

besides all the possible permutation of $\mathcal{V}, \mathcal{W}, \mathcal{Z}, \mathcal{T}$ except those which give redundant terms.

The set $S k$ : Representation for skew-symmetric second order tensorial functions.

- For every tensor: $\mathcal{V}, \mathcal{V}^{3}$,

- For every couple of tensors:

$\mathcal{W V}-\mathcal{V W}, \mathcal{V W} \mathcal{V}, \mathcal{W}^{3} \mathcal{V}-\mathcal{V} \mathcal{W}^{3}, \mathcal{V}^{2} \mathcal{W} \mathcal{V}-\mathcal{V} \mathcal{W} \mathcal{V}^{2}$ $\mathcal{W}^{2} \mathcal{V}^{2} \mathcal{W}+\mathcal{W} \mathcal{V}^{2} \mathcal{W}^{2}+\mathcal{V}^{2} \mathcal{W}^{3}+\mathcal{W}^{3} \mathcal{V}^{2}$ $\mathcal{W}^{2} \mathcal{V}^{2} \mathcal{W} \mathcal{V}-\mathcal{V} \mathcal{W} \mathcal{V}^{2} \mathcal{W}^{2}+\mathcal{V}^{2} \mathcal{W}^{3} \mathcal{V}-\mathcal{V} \mathcal{W}^{3} \mathcal{V}^{2}$ $\mathcal{V}^{2} \mathcal{W}^{2} \mathcal{V}^{2} \mathcal{W}+\mathcal{W} \mathcal{V}^{2} \mathcal{W}^{2} \mathcal{V}^{2}, \mathcal{V}^{2} \mathcal{W}^{2} \mathcal{V}^{2} \mathcal{W}-\mathcal{V} \mathcal{W} \mathcal{V}^{2} \mathcal{W}^{2} \mathcal{V}^{2}$ $\mathcal{V}^{2} \mathcal{W}+\mathcal{W} \mathcal{V}^{2}, \mathcal{V}^{2} \mathcal{W}^{2}-\mathcal{W}^{2} \mathcal{V}^{2}$

- For every set of three tensors: $\mathcal{W V U}+\mathcal{U V W}$,

besides all the possible permutation of $\mathcal{V}, \mathcal{W}, \mathcal{Z}$ except those which give redundant terms.

\section{Some useful properties for skew-symmetric second order tensors, in a n-dimensional space.}

In this paper we will use the following properties only for the case $n=4$; we will prove them for every $n$, because this general case is not more difficult to treat than the particular case. The proofs of these properties is shifted in the appendix. 
Property 1: "A skew-symmetric second order tensor $\mathcal{V}$ has only eigenvalues whose real part is zero; in particular, if $\mathcal{V}$ has a real eigenvalue, it is zero".

An immediate consequence of this property is that, if $n$ is odd, then $\mathcal{V}$ has surely the zero eigenvalue; this is because the characteristic equation is of degree $n$ which is odd and, consequently, has a real eigenvalue.

Property 2: "If $\vec{u}+i \vec{v}$ is an eigenvector of the skew-symmetric second order tensor $\mathcal{V}$ corresponding to the eigenvalue $a+i b$ with $b \neq 0$, then we have $a=0, \vec{u} \cdot \vec{v}=0, u^{2}=v^{2} \quad "$.

Property 3: "There is a basis of orthonormal vectors $\vec{u}_{1}, \vec{v}_{1}, \cdots, \vec{u}_{r}, \vec{v}_{r}$, $\vec{w}_{1}, \cdots, \vec{w}_{n-2 r}$ such that

$\vec{u}_{s}+i \vec{v}_{s}$ is an eigenvector of $\mathcal{V}$ corresponding to the eigenvalue $i b_{s}$ with $b_{s} \neq 0$ for $s=1, \cdots, r$, while

$\vec{w}_{p}$ for $p=1, \cdots, n-2 r$ is an eigenvector of $\mathcal{V}$ corresponding to the eigenvalue $\lambda=0$ ".

Property 4: "The tensor $\mathcal{V}$ has a number of linearly independent eigenvectors, corresponding to each eigenvalue $\lambda$, which is equal to the multiplicity of $\lambda "$.

Property 5: "If $\vec{u}+i \vec{v}$ is an eigenvector of $\mathcal{V}$ corresponding to the eigenvalue $i b$ with $b \neq 0$ and $\vec{w}$ is an eigenvector of $\mathcal{V}$ corresponding to the eigenvalue $\lambda=0$, then we have $\vec{w} \cdot \vec{u}=0$ and $\vec{w} \cdot \vec{v}=0 "$.

Property 6: "If $\vec{u}_{j}+i \vec{v}_{j}$, for $j=1,2$ are eigenvectors of $\mathcal{V}$ corresponding to the eigenvalues $i b_{j}$ with $0 \neq\left(b_{1}\right)^{2} \neq\left(b_{2}\right)^{2} \neq 0$, then we have $\vec{u}_{1} \cdot \vec{u}_{2}=0$, $\vec{u}_{1} \cdot \vec{v}_{2}=0, \vec{v}_{1} \cdot \vec{u}_{2}=0$ and $\vec{v}_{1} \cdot \vec{v}_{2}=0 "$.

Property 7: "If $\vec{u}_{j}+i \vec{v}_{j}$, for $j=1,2$ are eigenvectors of $\mathcal{V}$ corresponding to the same eigenvalue $i b$ with $b \neq 0$, then we have $\vec{u}_{1} \cdot \vec{u}_{2}=\vec{v}_{1} \cdot \vec{v}_{2}$, $\vec{u}_{1} \cdot \vec{v}_{2}=-\vec{v}_{1} \cdot \vec{u}_{2} "$

A consequence of these properties is that, in order to obtain the basis indicated in Property 3, we can proceed as follows: If there is the zero eigenvalue, let us take a number of linearly independent eigenvectors equal to the multiplicity $\mu_{0}$ of this eigenvalue. After that, we orthonormalize this set of vectors (for example, with Smith's method), so obtaining a set $S_{0}$ of $\mu_{0}$ orthonormal eigenvectors. For every eigenvalue $i b_{j}$ with $b_{j}>0$ (because for $b_{j}<0$ we obtain the complex conjugate of these and we are treating them both together), let us 
take a number of linearly independent eigenvectors equal to the multiplicity $\mu_{j}$ of this eigenvalue and let they be $\vec{u}_{j 1}+i \vec{v}_{j 1}, \vec{u}_{j 2}+i \vec{v}_{j 2}, \cdots, \vec{u}_{j \mu_{j}}+i \vec{v}_{j \mu_{j}}$. We use now a modified Smith's method. For Property 2, we have that $\vec{u}_{j 1}$ and $\vec{v}_{j 1}$ are orthogonal and have the same modulus; so, dividing them by their common modulus, we obtain two orthonormal vectors. For the sake of simplicity, let us call these new vectors still with the same name, $\vec{u}_{j 1}$ and $\vec{v}_{j 1}$. Obviously, the new $\vec{u}_{j 1}+i \vec{v}_{j 1}$ still remains an eigenvector corresponding to $i b_{j}$. For every complex number $\lambda$, we have that $\vec{u}_{j 2}+i \vec{v}_{j 2}+\lambda\left(\vec{u}_{j 1}+i \vec{v}_{j 1}\right)$ still remains an eigenvector corresponding to $i b_{j}$; we choose $\lambda$ such that this new vector is orthogonal to $\vec{u}_{j 1}$, that is,

$\lambda=-\vec{u}_{j 1} \cdot \vec{u}_{j 2}-i \vec{u}_{j 1} \cdot \vec{v}_{j 2}$, where we have used the fact that $\vec{u}_{j 1}$ and $\vec{v}_{j 1}$ are two orthonormal vectors.

But, for Property 7 , it follows that this value of $\lambda$ is also equal to $\lambda=-\vec{v}_{j 1} \cdot \vec{v}_{j 2}+$ $i \vec{v}_{j 1} \cdot \vec{u}_{j 2}$. A consequence of this fact is that the vector $\vec{u}_{j 2}+i \vec{v}_{j 2}+\lambda\left(\vec{u}_{j 1}+i \vec{v}_{j 1}\right)$ is orthogonal also to $\vec{v}_{j 1}$.

For the sake of simplicity, let us call these new vector $\vec{u}_{j 2}+i \vec{v}_{j 2}+\lambda\left(\vec{u}_{j 1}+i \vec{v}_{j 1}\right)$ still with the same name $\vec{u}_{j 2}+i \vec{v}_{j 2}$. We do the same thing with all the other eigenvectors up to $\vec{u}_{j \mu_{j}}+i \vec{v}_{j \mu_{j}}$. in this way we obtain the eigenvectors $\vec{u}_{j 1}+i \vec{v}_{j 1}$, $\vec{u}_{j 2}+i \vec{v}_{j 2}, \cdots, \vec{u}_{j \mu_{j}}+i \vec{v}_{j \mu_{j}}$ where $\vec{u}_{j 1}$ and $\vec{v}_{j 1}$ are two orthonormal vectors and $\vec{u}_{j 2}, \vec{v}_{j 2}, \cdots, \vec{u}_{j \mu_{j}}, \vec{v}_{j \mu_{j}}$ are all orthogonal to $\vec{u}_{j 1}$ and $\vec{v}_{j 1}$.

Let us repeat this procedure starting from $\vec{u}_{j 2}+i \vec{v}_{j 2}$, instead of $\vec{u}_{j 1}+i \vec{v}_{j 1}$, and so on. At the end, we find the eigenvectors $\vec{u}_{j 1}+i \vec{v}_{j 1}, \vec{u}_{j 2}+i \vec{v}_{j 2}, \cdots, \vec{u}_{j \mu_{j}}+i \vec{v}_{j \mu_{j}}$ where $\vec{u}_{j r}, \vec{v}_{j r}$ are a set of $\mu_{j}$ orthonormal vectors.

We do the same thing for every eigenvalue $i b_{j}$ with $b_{j}>0$. The union of all these vectors, in this way obtained, are an orthonormal basis, thanks to Property 6. In this way we have obtained the basis indicated in Property 3.

Let us begin now the main part of this paper; the first case which will be treated is expressed in terms of the scalars

$$
4 t r \mathcal{V}^{4}-\left(t r \mathcal{V}^{2}\right)^{2} \quad ; \quad 2 t r \mathcal{V}^{4}-\left(t r \mathcal{V}^{2}\right)^{2}
$$

\section{Case (1): There is a skew-symmetric tensor $\mathcal{V}$ such that the scalars (1) are different from zero.}

A consequence of our hypothesis is that $\mathcal{V}$ has not the zero eigenvalue, otherwise for the property 3 there is a reference frame such that 
$\mathcal{V}=\left(\begin{array}{cccc}0 & V^{12} & 0 & 0 \\ -V^{12} & 0 & 0 & 0 \\ 0 & 0 & 0 & 0 \\ 0 & 0 & 0 & 0\end{array}\right)$

from which it follows $\mathcal{V}^{2}=\operatorname{diag}\left(-\left(V^{12}\right)^{2},-\left(V^{12}\right)^{2}, 0,0\right)$ and the scalar $(1)_{2}$ would be zero. Therefore, $\mathcal{V}$ has the eigenvalues $i a$, $i b$ with $a>0, b>0$. For the property 3 , there is a reference frame such that

$\mathcal{V}=\left(\begin{array}{cccc}0 & a & 0 & 0 \\ -a & 0 & 0 & 0 \\ 0 & 0 & 0 & b \\ 0 & 0 & -b & 0\end{array}\right)$

from which it follows $\mathcal{V}^{2}=\operatorname{diag}\left(-a^{2},-a^{2},-b^{2},-b^{2}\right)$ and the scalar $(1)_{1}$ becomes $4\left(a^{2}-b^{2}\right)^{2}$; this must be different from zero, for our hypothesis, so that we have $a \neq b$.

For every other skew-symmetric tensor $\mathcal{W}$, from $\operatorname{tr} \mathcal{V} \mathcal{W}$ and $\operatorname{tr} \mathcal{V}^{3} \mathcal{W}$ we obtain $W^{12}$ and $W^{34}$. For the sequel, the following scalar will be useful

$$
\begin{array}{r}
8 \operatorname{tr}\left(\mathcal{W}^{2} \mathcal{V}^{2} \mathcal{W}^{2} \mathcal{V}^{2}\right)-2\left(\operatorname{tr} \mathcal{V}^{2}\right) \operatorname{tr}\left(\mathcal{W}^{2} \mathcal{V}^{2} \mathcal{W}^{2}+\mathcal{W}^{4} \mathcal{V}^{2}\right)+ \\
2\left(\operatorname{tr} \mathcal{V}^{4}\right)\left(\operatorname{tr} \mathcal{W}^{4}\right)-4\left[\operatorname{tr}\left(\mathcal{W}^{2} \mathcal{V}^{2}\right)\right]^{2}-\left(\operatorname{tr} \mathcal{V}^{4}\right)\left(\operatorname{tr} \mathcal{W}^{2}\right)^{2}+ \\
2\left(\operatorname{tr} \mathcal{W}^{2} \mathcal{V}^{2}\right)\left(\operatorname{tr} \mathcal{V}^{2}\right)\left(t r \mathcal{W}^{2}\right)
\end{array}
$$

\subsection{Subcase $(1,1)$ : There is a skew-symmetric tensor $\mathcal{W}$ such that the scalar (2) is different from zero.}

Let us define $\quad \mathcal{I}_{1}=\frac{1}{b^{2}-a^{2}}\left(\mathcal{V}^{2}+b^{2} \mathcal{I}\right)$ and $\quad \mathcal{I}_{2}=\frac{1}{a^{2}-b^{2}}\left(\mathcal{V}+a^{2} \mathcal{I}\right)$

from which it follows

$$
\mathcal{I}_{1}=\left(\begin{array}{cccc}
1 & 0 & 0 & 0 \\
0 & 1 & 0 & 0 \\
0 & 0 & 0 & 0 \\
0 & 0 & 0 & 0
\end{array}\right) ; \mathcal{I}_{2}=\left(\begin{array}{cccc}
0 & 0 & 0 & 0 \\
0 & 0 & 0 & 0 \\
0 & 0 & 1 & 0 \\
0 & 0 & 0 & 1
\end{array}\right)
$$

After that the scalar (2) becomes

$$
4 \operatorname{tr}\left(\mathcal{W}^{2} \mathcal{I}_{1} \mathcal{W}^{2} \mathcal{I}_{1}\right)-2\left(\operatorname{tr} \mathcal{W}^{2} \mathcal{I}_{1}\right)^{2}+4 \operatorname{tr}\left(\mathcal{W}^{2} \mathcal{I}_{2} \mathcal{W}^{2} \mathcal{I}_{2}\right)-2\left(\operatorname{tr} \mathcal{W}^{2} \mathcal{I}_{2}\right)^{2}
$$


But

$$
\begin{aligned}
& \mathcal{W}^{2} \mathcal{I}_{1}=\left(\begin{array}{cccc}
\left(W^{2}\right)^{11} & \left(W^{2}\right)^{12} & 0 & 0 \\
\left(W^{2}\right)^{12} & \left(W^{2}\right)^{22} & 0 & 0 \\
\left(W^{2}\right)^{13} & \left(W^{2}\right)^{23} & 0 & 0 \\
\left(W^{2}\right)^{14} & \left(W^{2}\right)^{24} & 0 & 0
\end{array}\right) ; \\
& \mathcal{W}^{2} \mathcal{I}_{2}=\left(\begin{array}{cccc}
0 & 0 & \left(W^{2}\right)^{13} & \left(W^{2}\right)^{14} \\
0 & 0 & \left(W^{2}\right)^{23} & \left(W^{2}\right)^{24} \\
0 & 0 & \left(W^{2}\right)^{33} & \left(W^{2}\right)^{34} \\
0 & 0 & \left(W^{2}\right)^{34} & \left(W^{2}\right)^{44}
\end{array}\right),
\end{aligned}
$$

so that the scalar (4) becomes

$$
\begin{aligned}
& 2\left[\left(W^{2}\right)^{11}-\left(W^{2}\right)^{22}\right]^{2}+8\left[\left(W^{2}\right)^{12}\right]^{2}+2\left[\left(W^{2}\right)^{33}-\left(W^{2}\right)^{44}\right]^{2}+ \\
& +8\left[\left(W^{2}\right)^{34}\right]^{2}= \\
& =2\left[-\left(W^{13}\right)^{2}-\left(W^{14}\right)^{2}+\left(W^{23}\right)^{2}+\left(W^{24}\right)^{2}\right]^{2}+8\left[\left(W^{2}\right)^{12}\right]^{2}+ \\
& +2\left[-\left(W^{13}\right)^{2}-\left(W^{23}\right)^{2}+\left(W^{14}\right)^{2}+\left(W^{24}\right)^{2}\right]^{2}+8\left[\left(W^{2}\right)^{34}\right]^{2}= \\
& =4\left[\left(W^{24}\right)^{2}-\left(W^{13}\right)^{2}\right]^{2}+4\left[\left(W^{23}\right)^{2}-\left(W^{14}\right)^{2}\right]^{2}+ \\
& +8\left(W^{13} W^{23}+W^{14} W^{24}\right)^{2}++8\left(W^{13} W^{14}+W^{23} W^{24}\right)^{2}
\end{aligned}
$$

and this scalar must be different from zero, for the hypothesis of this subcase. We look now for a rotation of the axis $x_{1}$ and $x_{2}$ with an angle $\vartheta$ and another rotation of the axis $x_{3}$ and $x_{4}$ with an angle $\varphi$ such that the components 14 and 23 of $\mathcal{W}$, in the new frame, are zero.

The matrix of this change of frame is

$$
P=\left(\begin{array}{cccc}
\cos \vartheta & \sin \vartheta & 0 & 0 \\
-\sin \vartheta & \cos \vartheta & 0 & 0 \\
0 & 0 & \cos \varphi & \sin \varphi \\
0 & 0 & -\sin \varphi & \cos \varphi
\end{array}\right)
$$

But we have $\mathcal{W}^{\prime}=P^{T} \mathcal{W} P$ so that our first requirement amounts to say that the transpose of the first column of $P$, multiplied by $\mathcal{W}$ and then by the fourth column of $P$ gives zero, that is,

$$
(\cos \vartheta-\sin \vartheta 00)\left(\begin{array}{cccc}
0 & W^{12} & W^{13} & W^{14} \\
-W^{12} & 0 & W^{23} & W^{24} \\
-W^{13} & -W^{23} & 0 & W^{34} \\
-W^{14} & -W^{24} & -W^{34} & 0
\end{array}\right)\left(\begin{array}{c}
0 \\
0 \\
\sin \varphi \\
\cos \varphi
\end{array}\right)=0
$$


Similarly, the requirement $\left(W^{\prime}\right)^{23}=0$ amounts to say that the transpose of the second column of $P$, multiplied by $\mathcal{W}$ and then by the third column of $P$ gives zero, that is,

$$
(\sin \vartheta \cos \vartheta 00)\left(\begin{array}{cccc}
0 & W^{12} & W^{13} & W^{14} \\
-W^{12} & 0 & W^{23} & W^{24} \\
-W^{13} & -W^{23} & 0 & W^{34} \\
-W^{14} & -W^{24} & -W^{34} & 0
\end{array}\right)\left(\begin{array}{c}
0 \\
0 \\
\cos \varphi \\
-\sin \varphi
\end{array}\right)=0 .
$$

These two equations may also be written as

$$
\begin{aligned}
& \left(\begin{array}{cc}
\cos \vartheta & -\sin \vartheta
\end{array}\right)\left(\begin{array}{ll}
W^{13} & W^{14} \\
W^{23} & W^{24}
\end{array}\right)\left(\begin{array}{c}
\sin \varphi \\
\cos \varphi
\end{array}\right)=0, \\
& \left(\begin{array}{ll}
\sin \vartheta & \cos \vartheta
\end{array}\right)\left(\begin{array}{ll}
W^{13} & W^{14} \\
W^{23} & W^{24}
\end{array}\right)\left(\begin{array}{cc}
0 & 1 \\
-1 & 0
\end{array}\right)\left(\begin{array}{c}
\sin \varphi \\
\cos \varphi
\end{array}\right)=0 .
\end{aligned}
$$

This is a system of two homogeneous equations in the two unknowns $\sin \varphi$ and $\cos \varphi$; these cannot be both zero, so it is necessary that the determinant of coefficients is zero. Vice versa, if this is zero, we can leave one equation of the system (because it is proportional to the other) and one equation of this type has surely a solution in the unknown $\varphi$. Consequently, $\vartheta$ has to be chosen such that the above mentioned determinant is zero, that is,

$$
\begin{aligned}
& \left|\begin{array}{cc}
W^{13} \cos \vartheta-W^{23} \sin \vartheta & W^{14} \cos \vartheta-W^{24} \sin \vartheta \\
-W^{14} \sin \vartheta-W^{24} \cos \vartheta & W^{13} \sin \vartheta+W^{23} \cos \vartheta
\end{array}\right|=0, \quad \text { or } \\
& {\left[\begin{array}{l}
\left.\left(W^{13}\right)^{2}+\left(W^{14}\right)^{2}-\left(W^{23}\right)^{2}-\left(W^{24}\right)^{2}\right] \sin \vartheta \cos \vartheta+ \\
\quad+\left(W^{13} W^{23}+W^{14} W^{24}\right)\left(\cos ^{2} \vartheta-\sin ^{2} \vartheta\right)=0
\end{array}\right.}
\end{aligned}
$$

and this equation has certainly solutions in the unknown $\vartheta$. In this way we have proved that a reference frame exists where $W^{14}=0, W^{23}=0$.

Thanks to this result, the hypothesis that the scalar (5) is different from zero becomes

$\left(W^{24}\right)^{2} \neq\left(W^{13}\right)^{2}$. Moreover, the matrix $\mathcal{W}^{2}$ becomes

$$
\mathcal{W}^{2}=\left(\begin{array}{cc}
-\left(W^{12}\right)^{2}-\left(W^{13}\right)^{2} & 0 \\
0 & -\left(W^{12}\right)^{2}-\left(W^{24}\right)^{2} \\
0 & -W^{12} W^{13}-W^{24} W^{34} \\
W^{12} W^{24}+W^{13} W^{34} &
\end{array}\right.
$$




$$
\begin{gathered}
0 \\
-W^{12} W^{13}-W^{24} W^{34} \\
-\left(W^{34}\right)^{2}-\left(W^{13}\right)^{2}
\end{gathered}
$$

0

$$
\begin{gathered}
W^{12} W^{24}+W^{13} W^{34} \\
0 \\
0 \\
-\left(W^{34}\right)^{2}-\left(W^{24}\right)^{2}
\end{gathered}
$$

We note that, if $(\bar{\vartheta}, \bar{\varphi})$ is a solution of eqs. (7) and (8), also $\left(\bar{\vartheta}+\frac{\pi}{2}, \bar{\varphi}+\frac{\pi}{2}\right)$ is a solution. We can choose the solution such that $\left(W^{24}\right)^{2}<\left(W^{13}\right)^{2}$. In fact, eq. (6) with $\left.\vartheta=\frac{\pi}{2}, \varphi=\frac{\pi}{2}\right)$ is $P=\left(\begin{array}{cccc}0 & 1 & 0 & 0 \\ -1 & 0 & 0 & 0 \\ 0 & 0 & 0 & 1 \\ 0 & 0 & -1 & 0\end{array}\right)$ so that $\mathcal{W} P$ is obtained from $\mathcal{W}$ exchanging the first with the second column and the third with the fourth column and, after that, changing sign to the new first column and to the new third column. Similarly, $P^{T} \mathcal{W} P$ is obtained from $\mathcal{W} P$ exchanging the first with the second raw and the third with the fourth raw and, after that, changing sign to the new first raw and to the new third raw. In this way, $W^{13}$ and $W^{24}$ exchange one another and the remaining part of $\mathcal{W}$ remains unchanged. We conclude that we can choose the reference frame so that

$$
\left(W^{24}\right)^{2}<\left(W^{13}\right)^{2}
$$

Now the scalar (2) is the same of (4) and (5); consequently, from this scalar in the present reference frame we obtain $\left[\left(W^{24}\right)^{2}-\left(W^{13}\right)^{2}\right]^{2}$ from which, thanks to $(9)$, we obtain $\left(W^{13}\right)^{2}-\left(W^{24}\right)^{2}$.

After that, from $\operatorname{tr} \mathcal{W}^{2}$ we obtain $\left(W^{13}\right)^{2}+\left(W^{24}\right)^{2}$; this, jointly with $\left(W^{13}\right)^{2}-$ $\left(W^{24}\right)^{2}$, gives $\left(W^{13}\right)^{2}$ and $\left(W^{24}\right)^{2}$. Finally, we note that from $\mathcal{I}_{1} \mathcal{W}^{2} \mathcal{I}_{1}, \mathcal{I}_{1}$, $\mathcal{I}_{2} \mathcal{W}^{2} \mathcal{I}_{2}, \mathcal{I}_{2}$ we can deduce $\mathcal{J}_{1}=\operatorname{diag}(1,0,0,0), \mathcal{J}_{2}=\operatorname{diag}(0,1,0,0), \mathcal{J}_{3}=$ $\operatorname{diag}(0,0,1,0), \mathcal{J}_{4}=\operatorname{diag}(0,0,0,1)$. With this in mind, we accomplish the following passages.

Now $\left(W^{13}\right)^{2}>\left(W^{24}\right)^{2} \geq 0$ implies $W^{13} \neq 0$; the reference frame can be chosen such that $W^{13}>0$; in fact, if we would have $W^{13}<0$, changing the versus of the axis 1 and 2, we will have $W^{13}>0$ still maintaining the sign of $V^{12}, V^{34}$, that is, $V^{12}=a>0, V^{34}=b>0$.

After that, we obtain $W^{13}$ from $\left(W^{13}\right)^{2}$ and $W^{24}$ from $\operatorname{tr}(\mathcal{V W} \mathcal{V W})$.

For every other skew-symmetric tensor $\mathcal{U}$ we obtain $U^{12}$ and $U^{34}$ from $\operatorname{tr}(\mathcal{V U})$ and $\operatorname{tr}\left(\mathcal{V}^{3} \mathcal{U}\right)$.

- If $W^{24} \neq 0$, from $\operatorname{tr}(\mathcal{W U}), \operatorname{tr}\left(\mathcal{W}^{3} \mathcal{U}\right), \operatorname{tr}\left(\mathcal{W}^{2} \mathcal{V}^{2} \mathcal{W}+\mathcal{V}^{2} \mathcal{W}^{3} \mathcal{U}\right), \operatorname{tr}\left(\mathcal{V}^{2} \mathcal{W}^{2} \mathcal{V}^{2}\right.$ $\mathcal{W U})$ we obtain $\operatorname{tr}\left(\mathcal{I}_{1} \mathcal{W}^{2} \mathcal{I}_{1} \mathcal{W U}\right)$ which jointly with $\operatorname{tr}(\mathcal{W U})$ gives $U^{13}$ and $U^{24}$. 
Similarly, from $\operatorname{tr} \mathcal{W}(\mathcal{V U}-\mathcal{U} \mathcal{V}), \operatorname{tr} \mathcal{W}^{3}(\mathcal{V U}-\mathcal{U V}), \operatorname{tr} \mathcal{V}^{2} \mathcal{W}^{2} \mathcal{V}^{2} \mathcal{W}(\mathcal{V U}-\mathcal{U} \mathcal{V})$, $\operatorname{tr}\left(\mathcal{W}^{2} \mathcal{V}^{2} \mathcal{W}+\mathcal{V}^{2} \mathcal{W}^{3}\right)(\mathcal{V U}-\mathcal{U V})$, we obtain $\operatorname{tr} \mathcal{I}_{1} \mathcal{W}^{2} \mathcal{I}_{1} \mathcal{W}(\mathcal{V U}-\mathcal{U} \mathcal{V})$ which jointly with $\operatorname{tr} \mathcal{W}(\mathcal{V U}-\mathcal{U} \mathcal{V})$ gives $(V U-U V)^{13}$ and $(V U-U V)^{24}$, that is, $U^{14} b+U^{23} a$ and $-U^{14} a-U^{23} b$; from these two last quantities we find finally $U^{14}$ and $U^{23}$.

- If $W^{24}=0$, from $\operatorname{tr}(\mathcal{W U})$ we find $U^{13}$; from $\operatorname{tr} \mathcal{W} \mathcal{U N} \mathcal{V}$ we find $U^{24}$.

From $\operatorname{tr} \mathcal{W}(\mathcal{V U}-\mathcal{U V})$ and $\operatorname{tr} \mathcal{W} \mathcal{V}(\mathcal{V U}-\mathcal{U V}) \mathcal{V}$ we find $(V U-U V)^{13}$ and $(V U-U V)^{24}$ from which, as before, $U^{14}$ and $U^{23}$.

We note that the scalars which we have used, also those employed before this subsection, are included in the set $S$ which we reported in the Introduction.

We have only omitted $t r \mathcal{W}^{4} \mathcal{V}^{2}$ because, by using the Hamilton-Cayley' s theorem, we see that it is a function of $\operatorname{tr} \mathcal{V}^{2}, \operatorname{tr} \mathcal{W}^{2}, \operatorname{tr} \mathcal{W}^{4}$ and $\operatorname{tr} \mathcal{W}^{2} \mathcal{V}^{2}$ which are already present in the table.

Regarding the skew-symmetric tensor valued functions, we see that they are linear combinations of those listed in the following table.

The table $1 T s k$

- For every tensor: $\mathcal{V}, \mathcal{V}^{3}$,

- For every couple of tensors: $\mathcal{V}^{2} \mathcal{W}^{2} \mathcal{V}^{2} \mathcal{W}+\mathcal{W} \mathcal{V}^{2} \mathcal{W}^{2} \mathcal{V}^{2}$

$\mathcal{W}^{2} \mathcal{V}^{2} \mathcal{W}+\mathcal{W} \mathcal{V}^{2} \mathcal{W}^{2}+\mathcal{V}^{2} \mathcal{W}^{3}+\mathcal{W}^{3} \mathcal{V}^{2}, \mathcal{V}^{2} \mathcal{W}-\mathcal{V} \mathcal{W} \mathcal{V}^{2}$ $\mathcal{W}-\mathcal{V} \mathcal{W}, \mathcal{V}^{2} \mathcal{W}^{2} \mathcal{V}^{2} \mathcal{W}-\mathcal{V} \mathcal{W} \mathcal{V}^{2} \mathcal{W}^{2} \mathcal{V}^{2}, \mathcal{W}^{3} \mathcal{V}-\mathcal{V} \mathcal{W}^{3}$ $\mathcal{W}^{2} \mathcal{V}^{2} \mathcal{W}-\mathcal{V} \mathcal{W} \mathcal{V}^{2} \mathcal{W}^{2}+\mathcal{V}^{2} \mathcal{W}^{3} \mathcal{V}-\mathcal{V} \mathcal{W}^{3} \mathcal{V}^{2}, \mathcal{V} \mathcal{W}$

In fact,

- If $W^{24} \neq 0$, from these tensors we can obtain

$$
\begin{array}{r}
\mathcal{V}, \mathcal{V}^{3}, \mathcal{W}, \mathcal{I}_{1} \mathcal{W}^{2} \mathcal{I}_{1} \mathcal{W}+ \\
\mathcal{W} \mathcal{I}_{1} \mathcal{W}^{2} \mathcal{I}_{1}, \mathcal{I}_{1} \mathcal{W} \mathcal{V}-\mathcal{V} \mathcal{W} \mathcal{I}_{1} \\
\mathcal{I}_{1} \mathcal{W}^{2} \mathcal{I}_{1} \mathcal{W}-\mathcal{V} \mathcal{W} \mathcal{I}_{1} \mathcal{W}^{2} \mathcal{I}_{1}
\end{array}
$$

After that, by writing $\phi^{i j}$ as a linear combination of the tensors (10), we have that the components 14 and 23 give a system for the determination of the coefficients of $(10)_{5,6}$ with determinant of coefficients $\left|\begin{array}{cc}W^{13} b & \left(W^{2}\right)^{11} W^{13} b \\ -W^{24} b & -\left(W^{2}\right)^{22} W^{24} b\end{array}\right|=W^{13} W^{24} b^{2}\left[\left(W^{2}\right)^{11}-\left(W^{2}\right)^{22}\right] \neq 0$, so that we can determine these coefficients.

We can now consider the components 13 and 24 obtaining a system for 
the determination of the coefficients of $(10)_{3,4}$ with determinant of coefficients $\left|\begin{array}{ll}W^{13} & -\left[\left(W^{12}\right)^{2}+\left(W^{13}\right)^{2}\right] W^{13} \\ W^{24} & -\left[\left(W^{12}\right)^{2}+\left(W^{24}\right)^{2}\right] W^{24}\end{array}\right| \neq 0$, so that we can determine these coefficients.

Finally, the components 12 and 34 allow to obtain the coefficients of $(10)_{1,2}$.

- If $W^{24}=0$, from the tensors of table $1 T s k$ we can obtain

$$
\mathcal{V}, \mathcal{V}^{3}, \mathcal{W}, \mathcal{V} \mathcal{V} \mathcal{V}, \mathcal{W} \mathcal{V}-\mathcal{V} \mathcal{W}, \mathcal{V}^{2} \mathcal{W} \mathcal{V}-\mathcal{V} \mathcal{W} \mathcal{V}^{2}
$$

After that, the components 23 and 14 give a system for the determination of the coefficients of (11) 5,6 with determinant of coefficients

$$
\left|\begin{array}{cc}
W^{13} a & -W^{13} a b^{2} \\
W^{13} b & -W^{13} a^{2} b
\end{array}\right|=a b\left(W^{13}\right)^{2}\left(b^{2}-a^{2}\right) \neq 0,
$$

so that we can determine these coefficients.

We can now consider the component 24 obtaining the coefficient of (11) 4 and, subsequently, the component 13 obtaining the coefficient of $(11)_{3}$. Finally, the components 12 and 34 allows to obtain the coefficients of $(11)_{1,2}$.

The elements of the table $1 T s k$ and those of the following table $1 T s y$ are included in the sets $S k$ and $S y$ respectively; this is the case also for subsequent partial tables even if we will omit to say it. More than that, the sets $S k$ and Sy are the union of all these partial tables.

Regarding the symmetric tensor valued functions, we see that they are linear combinations of those listed in the following table.

The table $1 T s y$

- $\mathcal{I}$,

- For every tensor: $\mathcal{V}^{2}$,

- For every couple of tensors: $\mathcal{W} \mathcal{V}+\mathcal{V W}, \mathcal{V}^{2} \mathcal{W V}+\mathcal{V} \mathcal{W} \mathcal{V}^{2}, \mathcal{V}^{2} \mathcal{W}-\mathcal{W} \mathcal{V}^{2}$, $\mathcal{V}^{2} \mathcal{W}^{2}+\mathcal{W}^{2} \mathcal{V}^{2}, \mathcal{V}^{2} \mathcal{W}^{2} \mathcal{V}^{2} \mathcal{W}-\mathcal{W} \mathcal{V}^{2} \mathcal{W}^{2} \mathcal{V}^{2}$ $\mathcal{W}^{2} \mathcal{V}^{2} \mathcal{W}-\mathcal{W} \mathcal{V}^{2} \mathcal{W}^{2}+\mathcal{V}^{2} \mathcal{W}^{3}-\mathcal{W}^{3} \mathcal{V}^{2}, \mathcal{V}^{2} \mathcal{W}^{2} \mathcal{V}^{3}-\mathcal{V}^{3} \mathcal{W}^{2} \mathcal{V}^{2}, \mathcal{V}^{2} \mathcal{W}^{2} \mathcal{V}^{2}$ $\mathcal{V}^{3} \mathcal{W} \mathcal{V}-\mathcal{V} \mathcal{W} \mathcal{V}^{3}$, 
besides all the possible permutation of $\mathcal{V}, \mathcal{W}$ except those which give redundant terms. In fact,

- If $W^{24} \neq 0$, from these tensors we can obtain

$$
\begin{aligned}
& \mathcal{W}+\mathcal{V} \mathcal{W}, \mathcal{V}^{2} \mathcal{W}+\mathcal{V} \mathcal{W} \mathcal{V}^{2}, \mathcal{V}^{2} \mathcal{W}-\mathcal{W} \mathcal{V}^{2} \\
& \mathcal{I}_{1} \mathcal{W}^{2} \mathcal{I}_{1} \mathcal{W}-\mathcal{W} \mathcal{I}_{1} \mathcal{W}^{2} \mathcal{I}_{1}, \mathcal{I}_{1} \mathcal{W}^{2} \mathcal{I}_{1} \mathcal{V}-\mathcal{V} \mathcal{I}_{1} \mathcal{W}^{2} \mathcal{I}_{1} \\
& \mathcal{I}_{2} \mathcal{W}^{2} \mathcal{I}_{2} \mathcal{V}-\mathcal{V} \mathcal{I}_{2} \mathcal{W}^{2} \mathcal{I}_{2}, \mathcal{I}_{1} \mathcal{W}^{2} \mathcal{I}_{1}, \mathcal{I}_{1}, \mathcal{I}_{2} \mathcal{W}^{2} \mathcal{I}_{2}, \mathcal{I}_{2}
\end{aligned}
$$

After that, by writing $\phi^{i j}$ as a linear combination of the tensors (12), we have that the components 14 and 23 give a system for the determination of the coefficients of $(12)_{1,2}$ with $\left|\begin{array}{cc}W^{13} b+W^{24} a & -W^{13} a^{2} b-W^{24} a b^{2} \\ -W^{13} a-W^{24} b & W^{24} a^{2} b+W^{13} a b^{2}\end{array}\right|=$ $a b\left(a^{2}-b^{2}\right)\left[\left(W^{24}\right)^{2}-\left(W^{13}\right)^{2}\right] \neq 0$ as determinant of coefficients, so that we can determine these coefficients.

We can now consider the components 13 and 24 obtaining a system for the determination of the coefficients of $(12)_{3,4}$ with determinant of coefficients $\left|\begin{array}{cc}\left(b^{2}-a^{2}\right) W^{13} & \left(W^{2}\right)^{11} W^{13} \\ \left(b^{2}-a^{2}\right) W^{24} & \left(W^{2}\right)^{22} W^{24}\end{array}\right|=\left(b^{2}-a^{2}\right) W^{13} W^{24}\left[\left(W^{13}\right)^{2}-\left(W^{24}\right)^{2}\right] \neq 0$, so that we can determine these coefficients.

After that, the component 12 allows to obtain the coefficients of $(12)_{5}$ and the component 34 the coefficients of $(12)_{6}$.

Finally, the components 11 and 22 give the coefficients of $(12)_{7,8}$ and the components 33 and 44 give those of $(12)_{9,10}$.

- If $W^{24}=0$, from the tensors of table $1 T$ sy we can obtain

$$
\begin{aligned}
& \mathcal{W}+\mathcal{V} \mathcal{W}, \mathcal{V}^{2} \mathcal{W} \mathcal{V}+\mathcal{V} \mathcal{W} \mathcal{V}^{2}, \mathcal{V}^{2} \mathcal{W}-\mathcal{W} \mathcal{V}^{2} \\
& \mathcal{V}^{3} \mathcal{W}-\mathcal{V} \mathcal{W} \mathcal{V}^{3}, \mathcal{I}_{1} \mathcal{W}^{2} \mathcal{I}_{1} \mathcal{V}-\mathcal{V} \mathcal{I}_{1} \mathcal{W}^{2} \mathcal{I}_{1} \\
& \mathcal{I}_{2} \mathcal{W}^{2} \mathcal{I}_{2} \mathcal{V}-\mathcal{V} \mathcal{I}_{2} \mathcal{W}^{2} \mathcal{I}_{2}, \mathcal{I}_{1} \mathcal{W}^{2} \mathcal{I}_{1}, \mathcal{I}_{1}, \mathcal{I}_{2} \mathcal{W}^{2} \mathcal{I}_{2}, \mathcal{I}_{2}
\end{aligned}
$$

After that, the components 14 and 23 give a system for the determination of the coefficients of $(13)_{1,2}$ with determinant of coefficients $a b\left(W^{13}\right)^{2}\left(b^{2}-\right.$ $\left.a^{2}\right) \neq 0$, so that we can determine these coefficients.

We can now consider the component 13 obtaining the coefficient of $(13)_{3}$ and, subsequently, the component 24 obtaining the coefficient of $(13)_{4}$.

After that, the components 12 and 34 allows to obtain the coefficients of $(13)_{5,6}$, respectively. Finally, the components 11 and 22 give the coefficients of $(13)_{7,8}$ while the components 33 and 44 give those of $(13)_{9,10}$. 
In the remaining subcases the scalar (2) is zero so that also the scalar (5) is zero, that is,

$$
\begin{aligned}
& \left(W^{24}\right)^{2}=\left(W^{13}\right)^{2},\left(W^{23}\right)^{2}=\left(W^{14}\right)^{2}, \\
& W^{13} W^{23}+W^{14} W^{24}=0, W^{13} W^{14}+W^{23} W^{24}=0 .
\end{aligned}
$$

For the sequel, the following scalar will be useful

$$
2 \operatorname{tr} \mathcal{I}_{1}(\mathcal{W} \mathcal{Z}+\mathcal{Z} \mathcal{W}) \mathcal{I}_{1}(\mathcal{W} \mathcal{Z}+\mathcal{Z} \mathcal{W})-\left[\operatorname{tr} \mathcal{I}_{1}(\mathcal{W} \mathcal{Z}+\mathcal{Z} \mathcal{W})\right]^{2}
$$

\subsection{Subcase $(1,2)$ : There are two skew-symmetric tensors $\mathcal{W}$ and $\mathcal{Z}$ such that the scalar (15) is different from zero.}

We note firstly that the scalar present in (15) can be obtained from

$$
\begin{aligned}
& \operatorname{tr} \mathcal{V}^{2}(\mathcal{W} \mathcal{Z}+\mathcal{Z} \mathcal{W}) \mathcal{V}^{2}(\mathcal{W} \mathcal{Z}+\mathcal{Z} \mathcal{W}), \operatorname{tr} \mathcal{V}^{2}(\mathcal{W} \mathcal{Z}+\mathcal{Z} \mathcal{W})^{2} \\
& \operatorname{tr}(\mathcal{W} \mathcal{Z}+\mathcal{Z} \mathcal{W})^{2}, \operatorname{tr} \mathcal{V}^{2}(\mathcal{W} \mathcal{Z}+\mathcal{Z} \mathcal{W}), \operatorname{tr}(\mathcal{W} \mathcal{Z}+\mathcal{Z} \mathcal{W})
\end{aligned}
$$

Now the tensor $\mathcal{I}_{1}(\mathcal{W Z}+\mathcal{Z} \mathcal{W}) \mathcal{I}_{1}$ is symmetric and only its components 11,12 , 21, 22 may be different from zero; consequently, with a suitable rotation of the axis 1 and 2, we obtain that its new component 12 is zero and, moreover,

$$
\left[\mathcal{I}_{1}(\mathcal{W Z}+\mathcal{Z} \mathcal{W}) \mathcal{I}_{1}\right]^{11} \leq\left[\mathcal{I}_{1}(\mathcal{W} \mathcal{Z}+\mathcal{Z W}) \mathcal{I}_{1}\right]^{22}
$$

After that, we note that a rotation of the axis 3 and 4 leaves $\mathcal{I}_{1}(\mathcal{W} \mathcal{Z}+\mathcal{Z} \mathcal{W}) \mathcal{I}_{1}$ unchanged; we use this opportunity so that $W^{14}=0$. After that, eq. $(14)_{2}$ gives $W^{23}=0$.

In this way, the condition $\left[\mathcal{I}_{1}(\mathcal{W} \mathcal{Z}+\mathcal{Z} \mathcal{W}) \mathcal{I}_{1}\right]^{12}=0$ becomes

$$
-W^{13} Z^{23}-Z^{14} W^{24}=0 .
$$

The condition that the scalar (15) is not zero, now becomes

$$
4\left(W^{24} Z^{24}-W^{13} Z^{13}\right)^{2} \neq 0, \quad \text { from which } W^{24} Z^{24} \neq W^{13} Z^{13} .
$$

$$
\text { This allows to rewrite }(17) \text { as } W^{13} Z^{13}>W^{24} Z^{24} \text {. }
$$

If at least one between $W^{13}$ and $W^{24}$ is zero then, for eq. (14) 1 , also the other would be zero and this would violate (19); consequently, we have that $W^{13} \neq 0$ and $W^{24} \neq 0$.

Similarly, if at least one between $Z^{13}$ and $Z^{24}$ is zero then, for eq. $(14)_{1}$ written 
with $\mathcal{Z}$ instead of $\mathcal{W}$, also the other would be zero and this would violate (19); consequently, we have that $Z^{13} \neq 0$ and $Z^{24} \neq 0$.

By changing the versus of both the axis 1 and 2 we have that the tensor $\mathcal{V}$ remains unchanged, and also the results until now obtained; we use this opportunity so that $W^{13}>0$. From eq. (14) 1 it follows that two eventualities are possible:

1. $W^{24}=W^{13}$. In this case, eq. (19) gives $Z^{24} \neq Z^{13}$; this result jointly with $(14)_{1}$ written with $\mathcal{Z}$ instead of $\mathcal{W}$, gives $Z^{24}=-Z^{13}$. After that, eq. (20) gives $Z^{13}>0$.

Eq. (14) 4 written with $\mathcal{Z}$ instead of $\mathcal{W}$, gives $Z^{14}-Z^{23}=0$, while eq. (18) gives $Z^{14}+Z^{23}=0$; in conclusion we have that $Z^{14}=0, Z^{23}=0$.

2. $W^{24}=-W^{13}$. In this case, eq. (19) gives $-Z^{24} \neq Z^{13}$; this result jointly with $(14)_{1}$ written with $\mathcal{Z}$ instead of $\mathcal{W}$, gives $Z^{24}=Z^{13}$. After that, eq. (20) gives $Z^{13}>0$.

Eq. $(14)_{4}$ written with $\mathcal{Z}$ instead of $\mathcal{W}$, gives $Z^{14}+Z^{23}=0$, while eq. (18) gives $Z^{14}-Z^{23}=0$; in conclusion we have that $Z^{14}=0, Z^{23}=0$.

In both cases we have that $Z^{13}>0, Z^{14}=0, Z^{23}=0, W^{13} Z^{24}-Z^{13} W^{24}=$ $\pm 2 W^{13} Z^{13} \neq 0$. Moreover, we have $W^{24}=W^{13}, Z^{24}=-Z^{13}$ in the first case and $W^{24}=-W^{13}, Z^{24}=Z^{13}$ in the second case.

Now from $\operatorname{tr} \mathcal{V} \mathcal{W}, \operatorname{tr} \mathcal{V}^{3} \mathcal{W}$ we obtain $W^{12}, W^{34}$; from $\operatorname{tr} \mathcal{V} \mathcal{Z}, \operatorname{tr} \mathcal{V}^{3} \mathcal{Z}$ we obtain $Z^{12}, Z^{34}$

from $t r \mathcal{W}^{2}$ and $(14)_{1}$ we obtain $\left(W^{13}\right)^{2}$ from which $W^{13}$.

From $\operatorname{tr} \mathcal{Z}^{2}$ and $(14)_{1}$ written with $\mathcal{Z}$ instead of $\mathcal{W}$, we obtain $\left(Z^{13}\right)^{2}$ from which $Z^{13}$.

From $\operatorname{tr} \mathcal{V W \mathcal { W }}$ we obtain now $W^{24}$ and, from $\operatorname{tr} \mathcal{V} \mathcal{Z} \mathcal{Z}$ we deduce $Z^{24}$; in this way, it is now clear what of the above mentioned case is occurring.

For every other tensor $\mathcal{U}$ we deduce now the values of $U^{12}, U^{34}$ from $\operatorname{tr} \mathcal{V U}$, $\operatorname{tr} \mathcal{V}^{3} \mathcal{U}$ and of $U^{13}, U^{24}$ from $\operatorname{tr} \mathcal{W U}, \operatorname{tr} \mathcal{Z U}$ (more precisely, we have a system for the determination of the unknowns $U^{13}, U^{24}$ whose determinant of coefficients is $\left.W^{13} Z^{24}-Z^{13} W^{24} \neq 0\right)$.

From $\operatorname{tr} \mathcal{W}(\mathcal{V U}-\mathcal{U V})$ and $\operatorname{tr} \mathcal{Z}(\mathcal{V U}-\mathcal{U V})$ we obtain respectively

$U^{23}\left(-b W^{24}+a W^{13}\right)+U^{14}\left(b W^{13}-a W^{24}\right) \quad, \quad U^{23}\left(-b Z^{24}+a Z^{13}\right)+$ $U^{14}\left(b Z^{13}-a Z^{24}\right)$;

from these we can deduce $U^{23}, U^{14}$ because the determinant of coefficients is $\left(a^{2}-b^{2}\right)\left(W^{24} Z^{13}-Z^{24} W^{13}\right) \neq 0$. We note that the scalars here used are included in the set $S$ reported in the Introduction.

Regarding the skew-symmetric tensorial functions, we see now that their are 
linear combinations of

$$
\mathcal{V}, \mathcal{V}^{3}, \mathcal{W}, \mathcal{Z}, \mathcal{W V}-\mathcal{V W}, \mathcal{Z} \mathcal{V}-\mathcal{V} \mathcal{Z}
$$

In fact, by writing $\phi^{i j}$ as a linear combination of these tensors, we have that the components 14 and 23 give a system for the determination of the coefficients of $(21)_{5,6}$ with determinant of coefficients $\left(a^{2}-b^{2}\right)\left(W^{13} Z^{24}-Z^{13} W^{24}\right) \neq 0$; similarly, the components 13 and 24 constitute a system for the determination of the coefficients of $(21)_{3,4}$ with determinant of coefficients $W^{13} Z^{24}-Z^{13} W^{24} \neq 0$. Finally, the components 12 and 34 allow to obtain the coefficients of $(21)_{1,2}$. We conclude that there is no need to insert a new table, because the tensors (21) are already present in table $1 T s k$.

Regarding the symmetric tensor valued functions, we see that they are linear combinations of

$$
\begin{aligned}
& \mathcal{W} \mathcal{V}+\mathcal{V W}, \mathcal{Z} \mathcal{V}+\mathcal{V} \mathcal{Z}, \mathcal{V}^{2} \mathcal{W}-\mathcal{W} \mathcal{V}^{2}, \mathcal{V}^{2} \mathcal{Z}-\mathcal{Z} \mathcal{V}^{2} \\
& \mathcal{I}_{1}(\mathcal{W Z}+\mathcal{Z}) \mathcal{I}_{1} \mathcal{V}-\mathcal{V} \mathcal{I}_{1}(\mathcal{W Z}+\mathcal{Z} \mathcal{W}) \mathcal{I}_{1} \\
& \mathcal{I}_{2}(\mathcal{W Z}+\mathcal{Z} \mathcal{W}) \mathcal{I}_{2} \mathcal{V}-\mathcal{V} \mathcal{I}_{2}(\mathcal{W Z}+\mathcal{Z} \mathcal{W}) \mathcal{I}_{2} \\
& \mathcal{I}_{1}(\mathcal{W Z}+\mathcal{Z} \mathcal{W}) \mathcal{I}_{1}, \mathcal{I}_{1}, \mathcal{I}_{2}(\mathcal{W Z}+\mathcal{Z} \mathcal{W}) \mathcal{I}_{2}, \mathcal{I}_{2}
\end{aligned}
$$

In fact, by writing $\phi^{i j}$ as a linear combination of these tensors, we have that the components 14 and 23 give a system for the determination of the coefficients of $(22)_{1,2}$ with determinant of coefficients $\left(a^{2}-b^{2}\right)\left(W^{13} Z^{24}-Z^{13} W^{24}\right) \neq 0$; similarly, the components 13 and 24 constitute a system for the determination of the coefficients of $(22)_{3,4}$ with determinant of coefficients

$\left(a^{2}-b^{2}\right)^{2}\left(W^{13} Z^{24}-Z^{13} W^{24}\right) \neq 0$. Finally, the components 12 and 34 allows to obtain the coefficients of $(22)_{5,6}$ respectively.

Finally, the components 11 and 22 give the coefficients of $(22)_{7,8}$ and the components 33 and 44 give those of $(22)_{9,10}$.

Taking into account the expressions of $\mathcal{I}_{1}, \mathcal{I}_{2}$, the tensors (22) become linear combinations of the tensors in table $1 T s y$ and of those in the following new table.

The table $2 T s y$

For every set of three tensors: $\mathcal{V}^{2}(\mathcal{W Z}+\mathcal{Z} \mathcal{W}) \mathcal{V}^{3}-\mathcal{V}^{3}(\mathcal{W Z}+\mathcal{Z} \mathcal{W}) \mathcal{V}^{2}, \mathcal{V}^{2}(\mathcal{W Z}+$ $\mathcal{Z W}) \mathcal{V}+(\mathcal{W Z}+\mathcal{Z W}) \mathcal{V}^{3}-\mathcal{V}^{3}(\mathcal{W Z}+\mathcal{Z W})-\mathcal{V}(\mathcal{W Z}+\mathcal{Z} \mathcal{W}) \mathcal{V}^{2},(\mathcal{W Z}+\mathcal{Z} \mathcal{W}) \mathcal{V}-$ $\mathcal{V}(\mathcal{W Z}+\mathcal{Z} \mathcal{W}), \mathcal{V}^{2}(\mathcal{W Z}+\mathcal{Z} \mathcal{W}) \mathcal{V}^{2}, \mathcal{V}^{2}(\mathcal{W Z}+\mathcal{Z} \mathcal{W})+(\mathcal{W Z}+\mathcal{Z} \mathcal{W}) \mathcal{V}^{2}$ besides all the possible permutation of $\mathcal{V}, \mathcal{W}, \mathcal{Z}$, except those which give redundant terms. 
In the subcases which remain to consider, the scalar (15) is zero; in other words, we will have $\left[(\mathcal{W Z}+\mathcal{Z W})^{11}-(\mathcal{W Z}+\mathcal{Z W})^{22}\right]^{2}+4\left[(\mathcal{W Z}+\mathcal{Z W})^{12}\right]^{2}=0$, or

$$
\begin{aligned}
& W^{13} Z^{13}+W^{14} Z^{14}=W^{23} Z^{23}+W^{24} Z^{24}, \\
& W^{13} Z^{23}+W^{14} Z^{24}+W^{24} Z^{14}+W^{23} Z^{13}=0 .
\end{aligned}
$$

for every couple of skew-symmetric tensors $\mathcal{V}$ and $\mathcal{W}$.

As before, from $\operatorname{tr} \mathcal{V} \mathcal{W}, t r \mathcal{V}^{3} \mathcal{W}$, we obtain $W^{12}$ and $W^{34}$ for every tensor $\mathcal{W}$; after that, from $t r \mathcal{W}^{2}$ we obtain the following quantity, which will be useful for the sequel

$$
\left(W^{13}\right)^{2}+\left(W^{14}\right)^{2}+\left(W^{23}\right)^{2}+\left(W^{24}\right)^{2} .
$$

\subsection{Subcase $(1,3)$ : There is a skew-symmetric tensor $\mathcal{W}$ such that the quantity (24) is different from zero.}

With a rotation of the axis 3 and 4 we obtain $W^{14}=0$, leaving unchanged $\mathcal{V}$, $W^{12}$ and $W^{34}$. After that, eq. $(14)_{2}$ gives $W^{23}=0$.

Moreover, from $(24)$ and $(14)_{1}$ we obtain $\left(W^{13}\right)^{2} \neq 0$. By eventually changing the versus of both the axis 1 and 2 we have that $W^{13}>0$, leaving unchanged all the previous results. Form $\left(W^{13}\right)^{2}$ we deduce $W^{13}$. After that, from $\operatorname{tr} \mathcal{V} \mathcal{W} \mathcal{V W}$ we deduce the value of $W^{24}$.

For every other tensor $\mathcal{U}$, from $\operatorname{tr} \mathcal{V} \mathcal{U}, \operatorname{tr} \mathcal{V}^{3} \mathcal{U}$ we obtain $U^{12}$ and $U^{34}$. From $\operatorname{tr} \mathcal{W U}$ we obtain $W^{13} U^{13}+W^{24} U^{24}$ which, jointly with $(23)_{1}$ written with $\mathcal{U}$ instead of $\mathcal{Z}$, gives $W^{13} U^{13}$ and $W^{24} U^{24}$ from which $U^{13}$ and $U^{24}$ can be deduced.

From $\operatorname{tr} \mathcal{W}(\mathcal{V U}-\mathcal{U V})$ we obtain $W^{13}\left(a U^{23}+b U^{14}\right)-W^{24}\left(a U^{14}+b U^{23}\right)$; but, from $(23)_{2}$ written with $\mathcal{U}$ instead of $\mathcal{Z}$, we have also $W^{13} U^{23}+W^{24} U^{14}=0$. So we have 2 equations for the determination of the two unknowns $U^{14}$ and $U^{23}$; the determinant of coefficients is

$$
\begin{gathered}
\left|\begin{array}{c}
W^{24} \\
W^{13} b-W^{24} a \\
W^{13} a-W^{24} b
\end{array}\right|= \\
=\left\{\begin{array}{ccc}
2(a-b)\left(W^{13}\right)^{2} & \text { if } & W^{24}=W^{13} \\
-2(a+b)\left(W^{13}\right)^{2} & \text { if } & W^{24}=-W^{13} .
\end{array}\right.
\end{gathered}
$$

In every case this determinant is not zero. (Take into account that $a>0$, $b>0$ so that $a+b>0$; we have used also eq. (14) 1$)$. In this way we obtain $U^{14}$ and $U^{23}$. We note now that the scalars here used are present in the set $S$. 
Regarding the tensor valued functions, we distinguish two subcases, one with $W^{24}=W^{13}$ and the other with $W^{24}=-W^{13}$. Let us begin with the first of these.

1. $W^{24}=W^{13}$. In this case, eq. (23) gives $Z^{24}=Z^{13}, Z^{23}=-Z^{14}$ for every tensor $\mathcal{Z}$.

After that, for every tensor $\phi^{i j}$, we note that through the transformation

$$
P=\left(\begin{array}{cccc}
0 & 1 & 0 & 0 \\
-1 & 0 & 0 & 0 \\
0 & 0 & 0 & 1 \\
0 & 0 & -1 & 0
\end{array}\right)
$$

it transforms itself according to the law

$$
\left(\begin{array}{llll}
\phi^{11} & \phi^{12} & \phi^{13} & \phi^{14} \\
\phi^{21} & \phi^{22} & \phi^{23} & \phi^{24} \\
\phi^{31} & \phi^{32} & \phi^{33} & \phi^{34} \\
\phi^{41} & \phi^{42} & \phi^{43} & \phi^{44}
\end{array}\right) \rightarrow\left(\begin{array}{cccc}
\phi^{22} & -\phi^{21} & \phi^{24} & -\phi^{23} \\
-\phi^{12} & \phi^{11} & -\phi^{14} & \phi^{13} \\
\phi^{42} & -\phi^{41} & \phi^{44} & -\phi^{43} \\
-\phi^{32} & \phi^{31} & -\phi^{34} & \phi^{33}
\end{array}\right)
$$

whose left hand side is $\phi$ and whose right hand side is $P^{T} \phi P$ (We note that $P$ is the rotation of the axis 1 and 2 with an angle $\frac{\pi}{2}$, and the rotation of the axis 3 and 4 with an angle $\frac{\pi}{2}$ ). The independent variables transform themselves like $\phi$; this allows us to see that they remain unchanged trough this transformation. Therefore, also $\phi$ must remain unchanged because it is a function of those variables. Consequently, if $\phi$ is symmetric, we have $\phi^{11}=\phi^{22}, \phi^{12}=0, \phi^{33}=\phi^{44}, \phi^{34}=0, \phi^{13}=\phi^{24}, \phi^{14}=-\phi^{23}$; while if $\phi$ is skew-symmetric, we have $\phi^{13}=\phi^{24}, \phi^{14}=-\phi^{23}$. After that it is easy to verify that every skew-symmetric tensor $\phi$ is a linear combination of the tensors

$$
\mathcal{V}, \mathcal{V}^{3}, \mathcal{W}, \mathcal{W} \mathcal{V}-\mathcal{V W}
$$

In fact, by writing $\phi^{i j}$ as a linear combination of these tensors, we have that the components 14 and 23 give two equations for the determination of only one unknown, the coefficient of $(26)_{4}$; but, for the relation $\phi^{14}=-\phi^{23}$, these equations are equivalent and allow to determinate the unknown. Similarly, the components 13 and 24 give two equations for the determination of only one unknown, the coefficient of $(26)_{3}$; but, for the relation $\phi^{13}=\phi^{24}$, these equations are equivalent and allow to determinate the unknown. 
Finally, the components 12 and 34 allows to obtain the coefficients of $(26)_{1,2}$ respectively.

We conclude that there is no need to insert a new table, because the tensors (26) are already present in table $1 T s k$.

Regarding the symmetric tensor valued functions, we see that they are linear combinations of

$$
\mathcal{I}, \mathcal{V}^{2}, \mathcal{V}^{2} \mathcal{W}-\mathcal{W} \mathcal{V}^{2}, \mathcal{W} \mathcal{V}+\mathcal{V} \mathcal{W}
$$

In fact, by writing $\phi^{i j}$ as a linear combination of these tensors, we have that the components 14 and 23 give two equivalent equations (because $\phi^{14}=-\phi^{23}$ ) which allow to determinate the coefficient of $(27)_{4}$ (We have also to take into account that $a+b \neq 0$ because $a>0, b>0)$. Similarly, the components 13 and 24 give two equivalent equations (because $\phi^{13}=$ $\left.\phi^{24}\right)$ which allow to determinate the coefficient of $(27)_{3}$. The components 12 and 34 give identities (because $\phi^{12}=0, \phi^{23}=0$ ). The components $11,22,33$ and 44 give four equations of which only two are independent and give the coefficients of $(27)_{1,2}$.

We conclude also here that there is no need to insert a new table, because the tensors (27) are already present in table $1 T$ sy.

2. $W^{24}=-W^{13}$. In this case, eq. (23) gives $Z^{24}=-Z^{13}, Z^{23}=Z^{14}$ for every tensor $\mathcal{Z}$.

After that, for every tensor $\phi^{i j}$, we note that through the transformation

$$
\begin{aligned}
P & =\left(\begin{array}{cccc}
0 & 1 & 0 & 0 \\
-1 & 0 & 0 & 0 \\
0 & 0 & 0 & -1 \\
0 & 0 & 1 & 0
\end{array}\right) \text { it transforms itself according to the law } \\
& \left(\begin{array}{cccc}
\phi^{11} & \phi^{12} & \phi^{13} & \phi^{14} \\
\phi^{21} & \phi^{22} & \phi^{23} & \phi^{24} \\
\phi^{31} & \phi^{32} & \phi^{33} & \phi^{34} \\
\phi^{41} & \phi^{42} & \phi^{43} & \phi^{44}
\end{array}\right) \rightarrow\left(\begin{array}{cccc}
\phi^{22} & -\phi^{21} & -\phi^{24} & \phi^{23} \\
-\phi^{12} & \phi^{11} & \phi^{14} & -\phi^{13} \\
-\phi^{42} & \phi^{41} & \phi^{44} & -\phi^{43} \\
\phi^{32} & -\phi^{31} & -\phi^{34} & \phi^{33}
\end{array}\right)
\end{aligned}
$$

whose left hand side is $\phi$ and whose right hand side is $P^{T} \phi P$ (We note that $P$ is the rotation of the axis 1 and 2 with an angle $\frac{\pi}{2}$, and the rotation of the axis 3 and 4 with an angle $-\frac{\pi}{2}$ ). The independent variables transform themselves like $\phi$; this allows us to see that they remain unchanged trough this transformation. Therefore, also $\phi$ must remain unchanged because it 
is a function of those variables. Consequently, if $\phi$ is symmetric, we have $\phi^{11}=\phi^{22}, \phi^{12}=0, \phi^{33}=\phi^{44}, \phi^{34}=0, \phi^{13}=-\phi^{24}, \phi^{14}=\phi^{23}$; while if $\phi$ is skew-symmetric, we have $\phi^{13}=-\phi^{24}, \phi^{14}=\phi^{23}$. After that it is easy to verify that every skew-symmetric tensor $\phi$ is a linear combination of the tensors (26), already used in the case $W^{24}=W^{13}$. In fact, by writing $\phi^{i j}$ as a linear combination of these tensors, we have that the components 14 and 23 give two equations for the determination of only one unknown, the coefficient of $(26)_{4}$; but, for the relation $\phi^{14}=\phi^{23}$, these equations are equivalent and allow to determinate the unknown. Similarly, the components 13 and 24 give two equations for the determination of only one unknown, the coefficient of $(26)_{3}$; but, for the relation $\phi^{13}=-\phi^{24}$, these equations are equivalent and allow to determinate the unknown.

Finally, the components 12 and 34 allows to obtain the coefficients of $(26)_{1,2}$ respectively.

We conclude that there is no need to insert a new table, because the tensors (26) are already present in table $1 T s k$.

Regarding the symmetric tensor valued functions, we see that they are linear combinations of the tensors (27), already used in the case $W^{24}=$ $W^{13}$. In fact, by writing $\phi^{i j}$ as a linear combination of these tensors, we have that the components 14 and 23 give two equivalent equations (because $\phi^{14}=\phi^{23}$ ) which allow to determinate the coefficient of $(27)_{4}$ (We have also to take into account that $a+b \neq 0$ because $a>0, b>$ $0)$. Similarly, the components 13 and 24 give two equivalent equations (because $\phi^{13}=-\phi^{24}$ ) which allow to determinate the coefficient of $(27)_{3}$. The components 12 and 34 give identities (because $\phi^{12}=0, \phi^{34}=0$ ). The components 11, 22, 33 and 44 give four equations of which only two are independent and give the coefficients of $(27)_{1,2}$.

Also here, we conclude that there is no need to insert a new table, because the tensors (27) are already present in table $1 T s y$.

\subsection{Subcase $(1,4)$ : For every skew-symmetric tensor $\mathcal{W}$ we have that the quantity (24) is zero.}

It follows that $W^{14}=0, W^{23}=0, W^{13}=0, W^{24}=0$. Moreover, every tensor $\mathcal{W}$ can be obtained from $\operatorname{tr} \mathcal{V} \mathcal{W}, \operatorname{tr} \mathcal{V}^{3} \mathcal{W}$ which are scalars already present in the set $S$.

Regarding the representation for second order tensors $\phi^{i j}$ we note that, by changing the versus of both the axis 1 and 2, the independent variables remain unchanged so that every function depending on them must also remain 
unchanged. Instead of this, for the transformation law of tensors, we have that $\phi^{13}, \phi^{14}, \phi^{23}, \phi^{24}$ transform themselves into $-\phi^{13},-\phi^{14},-\phi^{23},-\phi^{34}$ respectively. It follows that

$$
\phi^{13}=0, \phi^{14}=0, \phi^{23}=0, \phi^{24}=0 .
$$

It follows that every skew-symmetric tensor valued function is a linear combination of $\mathcal{V}$ and $\mathcal{V}^{3}$, which are already present in Table $1 T s k$.

Regarding the symmetric tensor valued functions, besides eq. (28), we have also

$$
\phi^{11}=\phi^{22}, \phi^{12}=0, \phi^{33}=\phi^{44}, \phi^{34}=0 .
$$

In fact, with the change of reference frame (25) we have that $\phi^{11}, \phi^{22}, \phi^{12}, \phi^{33}$, $\phi^{44}, \phi^{34}$ transform themselves in $\phi^{22}, \phi^{11},-\phi^{12}, \phi^{44}, \phi^{33},-\phi^{34}$ respectively; on the other hand, they must remain unchanged because the independent variables remain unchanged. Consequently, eq. (29) must hold.

After that, we find that every symmetric tensor valued function is a linear combination of $\mathcal{I}$ and $\mathcal{V}^{2}$, which are already present in Table 1 Tsy. This concludes the first case. Let us proceed with the new one. It is described in terms of the quantity

$$
\left[4 t r \mathcal{W}^{4}-\left(\operatorname{tr} \mathcal{W}^{2}\right)^{2}\right]\left[2 \operatorname{tr} \mathcal{W}^{4}-\left(\operatorname{tr} \mathcal{W}^{2}\right)^{2}\right]
$$

4. Case (2) : For every skew-symmetric tensor $\mathcal{W}$ we have that the scalar (30) is zero, but there is one of them $\mathcal{V}$ such that $(1)_{2}$ is zero and $t \boldsymbol{r} \mathcal{V}^{2}$ is different from zero.

We choose a reference frame such that $\mathcal{V}=\left(\begin{array}{cccc}0 & a & 0 & 0 \\ -a & 0 & 0 & 0 \\ 0 & 0 & 0 & b \\ 0 & 0 & -b & 0\end{array}\right)$ with $a \geq$ $b \geq 0$. After that, the conditions, that $(1)_{2}$ is zero and $t r \mathcal{V}^{2}$ is different from zero, become $a^{2} b^{2}=0, a^{2}+b^{2} \neq 0$. It follows that $b=0, a>0$, so that $\mathcal{V}=\left(\begin{array}{cccc}0 & a & 0 & 0 \\ -a & 0 & 0 & 0 \\ 0 & 0 & 0 & 0 \\ 0 & 0 & 0 & 0\end{array}\right)$ 
For every skew-symmetric tensor $\mathcal{W}$, we obtain $W^{12}$ from $\operatorname{tr} \mathcal{V} \mathcal{W}$. Let us define now

$$
\mathcal{I}_{1}=\frac{2}{t r \mathcal{V}^{2}} \mathcal{V}^{2} \quad \text { and } \quad \mathcal{I}_{2}=-\frac{2}{t r \mathcal{V}^{2}} \mathcal{V}^{2}+\mathcal{I}
$$

from which it follows (3) also in this case.

\subsection{Subcase $(2,1)$ : There is a skew-symmetric tensor $\mathcal{W}$ such that the scalar (2) is different from zero.}

Now the scalar (2) takes the form (4) and other passages of subsection $(1,1)$ can be repeated, in particular those starting with eq. (4) and ending with eq. (9); we avoid to copy them here, for the sake of brevity. The result is that we find a reference frame where $W^{14}=0, W^{23}=0$ and $\left(W^{24}\right)^{2} \leq\left(W^{13}\right)^{2}$; after that, the condition that the scalar (2) is different from zero becomes $\left(W^{13}\right)^{2}-\left(W^{24}\right)^{2}>0$ and we are able to find the value of this quantity. Now we begin the new passages, referring no more to those of the subcase $(1,1)$. From $t r \mathcal{V}^{2} \mathcal{W}^{2}$ (which is already present in the set $S$ ) we obtain $\left(W^{13}\right)^{2}+\left(W^{24}\right)^{2}$ which, together with $\left(W^{13}\right)^{2}-\left(W^{24}\right)^{2}$, gives $\left(W^{13}\right)^{2}$ and $\left(W^{24}\right)^{2}$. After that, from $\operatorname{tr} \mathcal{W}^{2}$ we deduce $\left(W^{34}\right)^{2}$.

Now $\left(W^{13}\right)^{2}>\left(W^{24}\right)^{2} \geq 0$ implies $W^{13} \neq 0$; the reference frame can be chosen such that $W^{13}>0$; in fact, if we would have $W^{13}<0$, changing the versus of the axis 1 and 2, we will have $W^{13}>0$ still maintaining the sign of $V^{12}$, that is, $V^{12}=a>0$.

After that, we obtain $W^{13}$ from $\left(W^{13}\right)^{2}$. Let us proceed by distinguishing two situations.

\subsection{Subcase $(2,1,1):$ We have that $W^{24} \neq 0$.}

The reference frame can be chosen such that $W^{24}>0$; in fact, if we would have $W^{24}<0$, changing the versus of the axis 4 , we will have $W^{24}>0$ still maintaining the sign of $V^{12}$ and of $W^{13}$. After that, we deduce the value of $W^{24}$ from $\left(W^{24}\right)^{2}$. From $\operatorname{tr} \mathcal{V} \mathcal{W}^{3}$ we deduce $W^{34}$. So all the components of the tensor $\mathcal{W}$ have been obtained.

For every other skew-symmetric tensor $\mathcal{U}$, we obtain $U^{12}$ from $\operatorname{tr} \mathcal{V U}$;

from $\operatorname{tr}(\mathcal{W U}), \operatorname{tr} \mathcal{V}^{2}(\mathcal{W U}+\mathcal{U} \mathcal{W}), \operatorname{tr}\left(\mathcal{W}^{3} \mathcal{U}\right), \operatorname{tr}\left(\mathcal{W}^{2} \mathcal{V}^{2} \mathcal{W} \mathcal{U}+\mathcal{V}^{2} \mathcal{W}^{3} \mathcal{U}\right), \operatorname{tr}\left(\mathcal{V}^{2} \mathcal{W}^{2}\right.$ $\left.\mathcal{V}^{2} \mathcal{W U}\right)$ we obtain $\operatorname{tr}\left(\mathcal{I}_{1} \mathcal{W}^{2} \mathcal{I}_{1} \mathcal{W U}\right)$ and $\operatorname{tr}\left(\mathcal{I}_{1} \mathcal{W U}\right)$; these two scalars, in turns, give $U^{13}$ and $U^{24}$.

Similarly, from $\operatorname{tr} \mathcal{W}(\mathcal{V U}-\mathcal{U} \mathcal{V}), \operatorname{tr} \mathcal{V}^{2} \mathcal{W}(\mathcal{V U}-\mathcal{U V}), \operatorname{tr} \mathcal{W}^{3}(\mathcal{V U}-\mathcal{U V}), \operatorname{tr} \mathcal{V}^{2} \mathcal{W}^{2}$ $\mathcal{V}^{2} \mathcal{W}(\mathcal{V U}-\mathcal{U} \mathcal{V}), \operatorname{tr}\left(\mathcal{W}^{2} \mathcal{V}^{2} \mathcal{W}+\mathcal{V}^{2} \mathcal{W}^{3}\right)(\mathcal{V U}-\mathcal{U} \mathcal{V})$, we obtain $\operatorname{tr} \mathcal{I}_{1} \mathcal{W}^{2} \mathcal{I}_{1} \mathcal{W}(\mathcal{V U}-$ 
$\mathcal{U V}), \operatorname{tr} \mathcal{I}_{1} \mathcal{W}(\mathcal{V U}-\mathcal{U V})$ from which $(V U-U V)^{13}$ and $(V U-U V)^{24}$; from these two last quantities we find finally $U^{14}$ and $U^{23}$. Finally, from $\operatorname{tr} \mathcal{W} \mathcal{V} \mathcal{W}$ we obtain $U^{34}$.

The scalars here used are present in the set $S$.

Regarding the skew-symmetric tensor valued functions, we see that they are linear combinations of those listed in the following table 3Tsk and of those in the previous table $1 T s k$. In fact, from these tensors we can obtain

$$
\begin{aligned}
& \mathcal{I}_{2} \mathcal{W V}-\mathcal{V W} \mathcal{I}_{2}, \mathcal{I}_{2} \mathcal{W}^{2} \mathcal{I}_{2} \mathcal{W V}-\mathcal{V} \mathcal{W} \mathcal{I}_{2} \mathcal{W}^{2} \mathcal{I}_{2}, \mathcal{W V W} \\
& \mathcal{I}_{1} \mathcal{W}+\mathcal{W} \mathcal{I}_{1}, \mathcal{I}_{1} \mathcal{W}^{2} \mathcal{I}_{1} \mathcal{W}+\mathcal{W} \mathcal{I}_{1} \mathcal{W}^{2} \mathcal{I}_{1}, \mathcal{V}
\end{aligned}
$$

After that, by writing $\phi^{i j}$ as a linear combination of these tensors, we have that the component 34 gives the coefficient of $(31)_{3}$; after that, the components 14 and 23 give a system for the determination of the coefficients of $(31)_{1,2}$ with determinant of coefficients

$$
\left|\begin{array}{cc}
-W^{24} a & -\left(W^{2}\right)^{44} W^{24} a \\
W^{13} a & \left(W^{2}\right)^{33} W^{13} a
\end{array}\right|=-W^{13} W^{24} a^{2}\left[\left(W^{2}\right)^{33}-\left(W^{2}\right)^{44}\right] \neq 0 \text {, so that we }
$$

can determine these coefficients.

We can now consider the components 13 and 24 obtaining a system for the determination of the coefficients of $(31)_{4,5}$ with determinant of coefficients $\left|\begin{array}{cc}W^{13} & \left(W^{2}\right)^{11} W^{13} \\ W^{24} & \left(W^{2}\right)^{22} W^{24}\end{array}\right|=W^{13} W^{24}\left[\left(W^{2}\right)^{22}-\left(W^{2}\right)^{11}\right] \neq 0$, so that we can determine these coefficients.

Finally, the component 12 allows to obtain the coefficient of $(31)_{6}$.

The table $3 T s k$

- For every couple of tensors: $\mathcal{V}^{2} \mathcal{W}+\mathcal{W} \mathcal{V}^{2}, \mathcal{V}^{2} \mathcal{W}^{2}-\mathcal{W}^{2} \mathcal{V}^{2}$,

- For every set of three tensors: $\mathcal{W V U}+\mathcal{U N} \mathcal{W}$,

Regarding the symmetric tensor valued functions, we see that they are linear combinations of those listed in the following table 3Tsy and of those in the previous table $1 T s y$. In fact, from these tensors we can obtain

$$
\begin{aligned}
& \mathcal{W} \mathcal{V} \mathcal{W}^{2} \mathcal{V}^{2} \mathcal{W}-\mathcal{W} \mathcal{V}^{2} \mathcal{W}^{2} \mathcal{V} \mathcal{W}, \mathcal{V}^{2} \mathcal{W}^{2} \mathcal{V}-\mathcal{V} \mathcal{W}^{2} \mathcal{V}^{2} \\
& \mathcal{V}^{2} \mathcal{W}-\mathcal{W} \mathcal{V}^{2}, \mathcal{V}^{2} \mathcal{W}^{2} \mathcal{V}^{2} \mathcal{W}-\mathcal{W} \mathcal{V}^{2} \mathcal{W}^{2} \mathcal{V}^{2}, \mathcal{W} \mathcal{V}+\mathcal{V} \mathcal{W} \\
& \mathcal{W} \mathcal{V} \mathcal{W}^{2} \mathcal{V}^{2}+\mathcal{V}^{2} \mathcal{W}^{2} \mathcal{V} \mathcal{W}, \mathcal{I}_{1} \mathcal{W}^{2} \mathcal{I}_{1}, \mathcal{I}_{1}, \mathcal{I}_{2} \mathcal{W}^{2} \mathcal{I}_{2}, \mathcal{I}_{2}
\end{aligned}
$$


After that, by writing $\phi^{i j}$ as a linear combination of these tensors, we have that the component 34 gives the coefficient of $(32)_{1}$; the components 14 and 23 give a system for the determination of the coefficients of $(32)_{5,6}$ with

$$
\left|\begin{array}{cc}
W^{24} a & \left(W^{2}\right)^{11} W^{24} a^{3} \\
-W^{13} a & -\left(W^{2}\right)^{22} W^{13} a^{3}
\end{array}\right|=-a^{4} W^{13} W^{24}\left[\left(W^{2}\right)^{22}-\left(W^{2}\right)^{11}\right] \neq 0
$$

as determinant of coefficients, so that we can determine these coefficients.

We can now consider the components 13 and 24 obtaining a system for the determination of the coefficients of $(32)_{3,4}$ with determinant of coefficients $\left|\begin{array}{cc}-a^{2} W^{13} & a^{4}\left(W^{2}\right)^{11} W^{13} \\ -a^{2} W^{24} & a^{4}\left(W^{2}\right)^{22} W^{24}\end{array}\right| \neq 0$, so that we can determine these coefficients.

After that, the component 12 allows to obtain the coefficient of $(32)_{2}$, the components 11 and 22 give the coefficients of $(32)_{7,8}$ and the components 33 and 44 give those of $(32)_{9,10}$.

The table $3 T$ sy

For every couple of tensors: $\mathcal{W} \mathcal{V} \mathcal{W}^{2} \mathcal{V}^{2} \mathcal{W}-\mathcal{W} \mathcal{V}^{2} \mathcal{W}^{2} \mathcal{V W}$

$$
\mathcal{W} \mathcal{V} \mathcal{W}^{2} \mathcal{V}^{2}+\mathcal{V}^{2} \mathcal{W}^{2} \mathcal{V} \mathcal{W}, \mathcal{V}^{2} \mathcal{W}^{2} \mathcal{V}-\mathcal{V} \mathcal{W}^{2} \mathcal{V}^{2}
$$

(We have taken into account that the term $\mathcal{W}^{2} \mathcal{V}^{3}-\mathcal{V}^{3} \mathcal{W}^{2}$ which is present in Table $1 T s y$, in the present case becomes $-a^{2}\left(\mathcal{W}^{2} \mathcal{V}-\mathcal{V} \mathcal{W}^{2}\right)$ because $\mathcal{V}^{3}=$ $\left.-a^{2} \mathcal{V}\right)$.

\subsection{Subcase $(2,1,2):$ We have that $W^{24}=0$.}

From $\operatorname{tr} \mathcal{W}^{2}$ we find $\left(W^{34}\right)^{2}$.

\subsection{Subcase $(2,1,2,1):$ We have that $\left(W^{34}\right)^{2} \neq 0$.}

The reference frame can be chosen such that $W^{34}>0$ and its value obtained from $\left(W^{34}\right)^{2}$; in fact, if we would have $W^{34}<0$, changing the versus of the axis 4 we will have $W^{34}>0$ still maintaining the sign of $V^{12}$ and of $W^{13}$. Up to now, the components of $\mathcal{W}$ are known.

For every other skew-symmetric tensor $\mathcal{U}$, from the scalars $\operatorname{tr} \mathcal{V U}, \operatorname{tr} \mathcal{V}^{2}(\mathcal{W U}+$ $\mathcal{U W}), \operatorname{tr} \mathcal{W U}, \operatorname{tr} \mathcal{W}(\mathcal{V U}-\mathcal{U V}), \operatorname{tr} \mathcal{V}^{2} \mathcal{W}^{2} \mathcal{U}, \operatorname{tr} \mathcal{W}^{2}(\mathcal{U V}+\mathcal{V U})$ we obtain $U^{12}, U^{13}$, $U^{34}, U^{23}, U^{14}, U^{24}$, respectively. So all the tensor $\mathcal{U}$ has been obtained. These scalars have been inserted in the set $S$. 
Regarding the skew-symmetric tensor valued functions, we see that they are linear combinations of those listed in the tables $1 T s k$ and $3 T s k$. In fact, from these tensors we can obtain $\mathcal{I}_{2} \mathcal{W V}-\mathcal{V} \mathcal{W} \mathcal{I}_{2}$,

$$
\mathcal{I}_{1} \mathcal{W}+\mathcal{W} \mathcal{I}_{1}, \mathcal{V}, \mathcal{W}, \mathcal{V}^{2} \mathcal{W}^{2}-\mathcal{W}^{2} \mathcal{V}^{2}, \mathcal{V} \mathcal{W}^{2}+\mathcal{W}^{2} \mathcal{V}
$$

After that, by writing $\phi^{i j}$ as a linear combination of these tensors, we have that the components 24, 14, 34, 23, 13, 12 taken in this order, give the coefficients of $(33)_{6},(33)_{5},(33)_{4},(33)_{1},(33)_{2},(33)_{3}$ respectively.

Regarding the symmetric tensor valued functions, we see that they are linear combinations of the following tensors which, in turn, are linear combinations of those listed in the tables $1,2,3 T$ sy.

$$
\begin{gathered}
\mathcal{W} \mathcal{V}+\mathcal{V} \mathcal{W}, \mathcal{V}^{2} \mathcal{W}-\mathcal{W} \mathcal{V}^{2}, \mathcal{V}^{2} \mathcal{W}^{2} \mathcal{V}-\mathcal{V} \mathcal{W}^{2} \mathcal{V}^{2}, \mathcal{I}_{1} \mathcal{W}^{2} \mathcal{I}_{1}, \mathcal{I}_{1} \\
\mathcal{I}_{2} \mathcal{W}^{2} \mathcal{I}_{2}, \mathcal{I}_{2}, \mathcal{W}^{2}, \mathcal{W}^{2} \mathcal{V}-\mathcal{V} \mathcal{W}^{2}, \mathcal{I}_{2} \mathcal{W}^{2} \mathcal{I}_{2} \mathcal{W}-\mathcal{W} \mathcal{I}_{2} \mathcal{W}^{2} \mathcal{I}_{2}
\end{gathered}
$$

In fact, by writing $\phi^{i j}$ as a linear combination of these tensors, we have that the components $34,24,14,23,13,12$ taken in this order, give the coefficients of $(34)_{10},(34)_{9},(34)_{8},(34)_{1},(34)_{2},(34)_{3}$ respectively; finally, the components 11 and 22 give the coefficients of $(34)_{4,5}$ and the components 33 and 44 give those of $(34)_{6,7}$.

\subsection{Subcase $(2,1,2,2):$ We have that $\left(W^{34}\right)^{2}=0$.}

Up to now, the components of $\mathcal{W}$ are known. For every other skew-symmetric tensor $\mathcal{U}$, from the scalars $\operatorname{tr} \mathcal{V} \mathcal{U}, \operatorname{tr} \mathcal{V}^{2}(\mathcal{W U}+\mathcal{U} \mathcal{W}), \operatorname{tr} \mathcal{W}(\mathcal{V U}-\mathcal{U} \mathcal{V})$ we obtain $U^{12}, U^{13}, U^{23}$. After that, from $\operatorname{tr} \mathcal{V}^{2} \mathcal{U}^{2}, \operatorname{tr} \mathcal{V}^{2} \mathcal{W}^{2} \mathcal{V}^{2} \mathcal{U}^{2}$ we obtain $\left(U^{14}\right)^{2}$ and $\left(U^{24}\right)^{2}$; moreover, from $\operatorname{tr} \mathcal{U}^{2}$ we obtain $\left(U^{34}\right)^{2}$.

\subsection{Subcase $(2,1,2,2,1):$ There is a skew-symmetric tensor $\mathcal{U}$ such that $\left(U^{14}\right)^{2} \neq 0$.}

The reference frame can be chosen such that $U^{14}>0$; in fact, if we would have $U^{14}<0$, changing the versus of the axis 4 , we will have $U^{14}>0$ still maintaining the sign of $V^{12}$ and of $W^{13}$. After that, we deduce the value of $U^{14}$ from $\left(U^{14}\right)^{2}$. From $\operatorname{tr} \mathcal{V}^{2} \mathcal{W} \mathcal{U}^{2}$ we deduce now $U^{34}$ and, from $\operatorname{tr} \mathcal{V} \mathcal{W}^{2} \mathcal{V}^{2} \mathcal{U}^{2}$ we obtain $U^{24}$. So all the tensor $\mathcal{U}$ is known. For every other skew-symmetric tensor $\mathcal{Z}$, we already know $Z^{12}, Z^{13}, Z^{23}$ as we have already done with $\mathcal{U}$.

Now we note that, from $\operatorname{tr} \mathcal{Z}(\mathcal{V U}-\mathcal{U} \mathcal{V})$ we obtain $(U Z)^{12}-(U Z)^{21}$; thanks to 
this, $\operatorname{tr} \mathcal{V}^{2} \mathcal{W U Z}$ gives $(U Z)^{13}$ because $W^{12}\left[(U Z)^{12}-(U Z)^{21}\right]$ is already known. From $(U Z)^{13}$ we obtain $Z^{34}$.

Now, from $\operatorname{tr} \mathcal{U}, \operatorname{tr} \mathcal{V}^{2} \mathcal{W}^{2} \mathcal{V}^{2} \mathcal{U Z}$ we obtain $U^{14} Z^{14}$ and $U^{24} Z^{24}$; from the first one of these quantities we obtain $Z^{14}$.

Finally, from $(U Z)^{12}-(U Z)^{21}$ (which we already know), we deduce $Z^{24}$. The scalars here used are already present in the set $S$.

Regarding the skew-symmetric tensor valued functions, we see that they are linear combinations of those listed in the tables $1 T s k$ and $3 T s k$. In fact, from these tensors we can obtain $\mathcal{I}_{2} \mathcal{W V}-\mathcal{V} \mathcal{W} \mathcal{I}_{2}$,

$$
\mathcal{I}_{1} \mathcal{W}+\mathcal{W I}_{1}, \mathcal{V}, \mathcal{V}^{2} \mathcal{U}+\mathcal{U} \mathcal{V}^{2}, \mathcal{U} \mathcal{V}-\mathcal{V U}, \mathcal{W U}-\mathcal{U} \mathcal{W}
$$

After that, by writing $\phi^{i j}$ as a linear combination of these tensors, we have that the component 34 gives the coefficient of $(35)_{6}$; after that, the components 14 and 24 give a system for the determination of the coefficients of $(35)_{4,5}$ with determinant of coefficients

$\left|\begin{array}{cc}-U^{14} a^{2} & -U^{24} a \\ -U^{24} a^{2} & U^{14} a\end{array}\right|=-a^{3}\left[\left(U^{14}\right)^{2}+\left(U^{24}\right)^{2}\right] \neq 0$, so that we can determine these coefficients.

After that, the components 13, 23 and 12 give the coefficients of $(35)_{2},(35)_{1}$ and $(35)_{3}$ respectively.

Regarding the symmetric tensor valued functions, we see that they are linear combinations of those listed in the tables 1,2,3 Tsy. In fact, from these tensors we can obtain

$$
\begin{array}{r}
\mathcal{W V}+\mathcal{V W}, \mathcal{V}^{2} \mathcal{W}-\mathcal{W} \mathcal{V}^{2}, \mathcal{V}^{2} \mathcal{W}^{2} \mathcal{V}-\mathcal{V} \mathcal{W}^{2} \mathcal{V}^{2}, \mathcal{I}_{1} \mathcal{W}^{2} \mathcal{I}_{1}, \mathcal{I}_{1} \\
\mathcal{I}_{2} \mathcal{W}^{2} \mathcal{I}_{2}, \mathcal{I}_{2}, \mathcal{V}^{2} \mathcal{U}-\mathcal{U} \mathcal{V}^{2}, \mathcal{U} \mathcal{V}+\mathcal{V U}, \mathcal{W U}+\mathcal{U} \mathcal{W}
\end{array}
$$

After that, by writing $\phi^{i j}$ as a linear combination of these tensors, we have that the component 34 gives the coefficient of $(36)_{10}$; the components 14 and 24 give a system for the determination of the coefficients of $(36)_{8,9}$ with

$$
\left|\begin{array}{cc}
-U^{14} a^{2} & U^{24} a \\
-U^{24} a^{2} & -U^{14} a
\end{array}\right|=a^{3}\left[\left(U^{14}\right)^{2}+\left(U^{24}\right)^{2}\right] \neq 0
$$

as determinant of coefficients, so that we can determine these coefficients. After that, the components 13,23 and 12 give the coefficients of $(36)_{2},(36)_{1}$ and $(36)_{3}$ respectively.

Finally, the components 11 and 22 give the coefficients of $(36)_{4,5}$ and the components 33 and 44 give those of $(36)_{6,7}$. 


\subsection{Subcase $(2,1,2,2,2)$ : For every skew-symmetric tensor $\mathcal{U}$ we have $\left(U^{14}\right)^{2}=0$, but there is one of them, $\mathcal{U}$, such that} $\left(U^{24}\right)^{2} \neq 0$.

The reference frame can be chosen such that $U^{24}>0$; in fact, if we would have $U^{24}<0$, changing the versus of the axis 4 , we will have $U^{24}>0$ still maintaining the sign of $V^{12}$ and of $W^{13}$. After that, we deduce the value of $U^{24}$ from $\left(U^{24}\right)^{2}$. From $\operatorname{trU}^{2}(\mathcal{V} \mathcal{W}+\mathcal{W} \mathcal{V})$ we deduce now $U^{34}$. So all the tensor $\mathcal{U}$ is known. For every other skew-symmetric tensor $\mathcal{Z}$, we already know $Z^{12}$, $Z^{13}, Z^{23}$ as we have already done previously with $\mathcal{U}$. But we have also $Z^{14}=0$ because we are just in the subcase $U^{14}=0 \forall \mathcal{U}$.

From $\operatorname{tr} \mathcal{V}^{2}(\mathcal{U Z}+\mathcal{Z} \mathcal{U})$ we deduce now $Z^{24}$. Finally, from $\operatorname{tr} \mathcal{U} \mathcal{V} \mathcal{W}$ we obtain $Z^{34}$. The scalars here used are present in the set $S$.

Regarding the skew-symmetric tensor valued functions, we do the same passages of Subcase $(2,1,2,2,1)$, but with $\mathcal{W} \mathcal{U}+\mathcal{U N} \mathcal{W}$ instead of $\mathcal{W U}-\mathcal{U W}$ in (35). Regarding the symmetric tensor valued functions, we do the same passages of Subcase $(2,1,2,2,1)$, but with $(\mathcal{W} \mathcal{V}+\mathcal{V} \mathcal{W}) \mathcal{U}-\mathcal{U}(\mathcal{W} \mathcal{V}+\mathcal{V} \mathcal{W})$ instead of $\mathcal{W} \mathcal{U}+\mathcal{U}$ in $(36)$.

\subsection{Subcase $(2,1,2,2,3)$ : For every skew-symmetric tensor $\mathcal{U}$ we have $\left(U^{14}\right)^{2}=0,\left(U^{24}\right)^{2}=0$, but there is one of them, $\mathcal{U}$, such} that $\left(U^{34}\right)^{2} \neq 0$.

The reference frame can be chosen such that $U^{34}>0$; in fact, if we would have $U^{34}<0$, changing the versus of the axis 4 , we will have $U^{34}>0$ still maintaining the sign of $V^{12}$ and of $W^{13}$. After that, we deduce the value of $U^{34}$ from $\left(U^{34}\right)^{2}$. For every other skew-symmetric tensor $\mathcal{Z}$, we already know $Z^{12}, Z^{13}, Z^{23}$ as we have already done previously with $\mathcal{U}$. But we have also $Z^{14}=0, Z^{24}=0$ because we are just in the subcase $U^{14}=0, U^{24}=0 \forall \mathcal{U}$.

From $\operatorname{tr} \mathcal{U} \mathcal{Z}$ we deduce now $Z^{34}$. The scalars here used belong all to the set $S$. Regarding the skew-symmetric tensor valued functions, we do the same passages of Subcase $(2,1,2,2,1)$, but with $\mathcal{W U}-\mathcal{U W}, \mathcal{V} \mathcal{W}+\mathcal{U} \mathcal{W}, \mathcal{U}$ instead of $\mathcal{V}^{2} \mathcal{U}+$ $\mathcal{U} \mathcal{V}^{2}, \mathcal{U V}-\mathcal{V U}, \mathcal{W U}-\mathcal{U W}$ respectively in (35); moreover, it is easier to evaluate firstly the component 34 , then the component 14 and, after that, the component 24; for the other components we may proceed as in Subcase $(2,1,2,2,1)$.

Regarding the symmetric tensor valued functions, we do the same passages of Subcase $(2,1,2,2,1)$, but with $\mathcal{W U}+\mathcal{U} \mathcal{W},(\mathcal{V W}+\mathcal{W} \mathcal{V}) \mathcal{U}-\mathcal{U}(\mathcal{V W}+\mathcal{W} \mathcal{V})$, $\mathcal{W}^{2} \mathcal{U}-\mathcal{U} \mathcal{W}^{2}$ instead of $\mathcal{V}^{2} \mathcal{U}-\mathcal{U} \mathcal{V}^{2}, \mathcal{U V}+\mathcal{V U}, \mathcal{W U}+\mathcal{U} \mathcal{W}$ in (36) respectively; moreover, it is easier to evaluate firstly the component 34 , then the component 
24 and, after that, the component 14; for the other components we may proceed as in Subcase $(2,1,2,2,1)$.

\subsection{Subcase $(2,1,2,2,4)$ : For every skew-symmetric tensor $\mathcal{U}$ we have $\left(U^{14}\right)^{2}=0,\left(U^{24}\right)^{2}=0,\left(U^{34}\right)^{2}=0$.}

In this subcase all the tensors have been already deduced from scalars inserted in the set $S$.

Regarding the representation for second order tensors $\phi^{i j}$, both symmetric and skew-symmetric, we note that by changing the versus of the axis 4 the independent variables remain unchanged so that every function depending on them must also remain unchanged. Instead of this, for the transformation law of tensors, we have that $\phi^{14}, \phi^{24}, \phi^{34}$ change sign. It follows that $\phi^{14}=0, \phi^{24}=0$, $\phi^{34}=0$. Consequently, every symmetric tensor valued function is a linear combination of the tensors $(36)_{1-7}$ and every skew-symmetric tensor valued function is a linear combination of the tensors $(35)_{1,2,3}$.

In this way we have finished the subcases of Subcase $(2,1)$. In the other subcases of case (2) which remain to consider, the scalar (2) is zero so that also the scalar (5) is zero, that is, eqs. (14) hold. Even if they were written for the case (1), they hold also in the case (2). Similarly, the scalar (15) which was there introduced, can be used also now.

\subsection{Subcase $(2,2):$ There are two skew-symmetric tensors $\mathcal{W}$ and $\mathcal{Z}$ such that the scalar (15) is different from zero.}

We can repeat the same passages of the Subcase $(1,2)$ up to the inequality $(20)$. We avoid to copy them here, for the sake of brevity. After that we proceed as follows.

If at least one between $W^{13}$ and $W^{24}$ is zero then, for eq. (14) 1 , also the other would be zero and this would violate (19); consequently, we have that $W^{13} \neq 0$ and $W^{24} \neq 0$.

Similarly, if at least one between $Z^{13}$ and $Z^{24}$ is zero then, for eq. $(14)_{1}$ written with $\mathcal{Z}$ instead of $\mathcal{W}$, also the other would be zero and this would violate (19); consequently, we have that $Z^{13} \neq 0$ and $Z^{24} \neq 0$.

By changing the versus of both the axis 1 and 2 we have that the tensor $\mathcal{V}$ remains unchanged, and also the results until now obtained; we use this opportunity so that $W^{13}>0$.

We note that, by changing the versus of the axis $4, V^{12}$ and $W^{13}$ remain un- 
changed ( In subsection $(1,2)$ there was the component $V^{34}=b$ which changes sign with this inversion of the axis 4 ; but here we have $V^{34}=0$ so that it remains unchanged). We choose the versus of the axis 4 so that $W^{24}>0$; consequently, we now have $W^{24}=W^{13}$.

After that, eq. (19) gives $Z^{24} \neq Z^{13}$; this result jointly with (14) written with $\mathcal{Z}$ instead of $\mathcal{W}$, gives $Z^{24}=-Z^{13}$. After that, eq. (20) gives $Z^{13}>0$.

Eq. $(14)_{4}$ written with $\mathcal{Z}$ instead of $\mathcal{W}$, gives $Z^{14}-Z^{23}=0$, while eq gives $Z^{14}+Z^{23}=0$; in conclusion we have that $Z^{14}=0, Z^{23}=0$.

Now from $\operatorname{tr} \mathcal{V W}, \operatorname{tr} \mathcal{V} \mathcal{Z}$ we obtain $W^{12}, Z^{12}$. From $\operatorname{tr} \mathcal{I}_{1} \mathcal{W}^{2}, \operatorname{tr} \mathcal{I}_{1} \mathcal{Z}^{2}$, thanks also to $\left(W^{13}\right)^{2}=\left(W^{24}\right)^{2}$ and $\left(Z^{13}\right)^{2}=\left(Z^{24}\right)^{2}$, we find $\left(W^{13}\right)^{2}$ and $\left(Z^{13}\right)^{2}$ from which $W^{13}$ and $Z^{13}$. Consequently, we know also $W^{24}$ and $Z^{24}$.

From $\operatorname{tr} \mathcal{V} \mathcal{W}^{3}$ we obtain $W^{34}$ and from $\operatorname{tr} \mathcal{V} \mathcal{Z}^{3}$ we obtain $Z^{34}$.

For every other tensor $\mathcal{U}$ we deduce now the values of $U^{12}$ from $\operatorname{tr} \mathcal{V} \mathcal{U}$, the values of $U^{13}$ and $U^{24}$ from $\operatorname{tr} \mathcal{V}^{2}(\mathcal{W U}+\mathcal{U W})$ and

$\operatorname{tr} \mathcal{V}^{2}(\mathcal{Z U}+\mathcal{U Z})$. From $\operatorname{tr} \mathcal{W}(\mathcal{V U}-\mathcal{U} \mathcal{V})$ and $\operatorname{tr} \mathcal{Z}(\mathcal{V U}-\mathcal{U V})$ we obtain $U^{23}$ and $U^{14}$.

Finally, from $\operatorname{tr} \mathcal{V} \mathcal{W U} \mathcal{W}$ we obtain $U^{34}$. All the scalars here used are already present in the set $S$.

Regarding the skew-symmetric tensorial functions, we see now that their are linear combinations of

$$
\mathcal{V}, \mathcal{W} \mathcal{V} \mathcal{W}, \mathcal{V}^{2} \mathcal{W}+\mathcal{W} \mathcal{V}^{2}, \mathcal{V}^{2} \mathcal{Z}+\mathcal{Z} \mathcal{V}^{2}, \mathcal{W} \mathcal{V}-\mathcal{V} \mathcal{W}, \mathcal{Z} \mathcal{V}-\mathcal{V} \mathcal{Z}
$$

In fact, by writing $\phi^{i j}$ as a linear combination of these tensors, we have that the component 34 gives the coefficient of $(37)_{2}$, the components 14 and 23 give those of $(37)_{5,6}$, the components 13 and 24 those of $(37)_{3,4}$, the component 12 gives the coefficients of $(37)_{1}$. All the tensors (37) are already present in the previous tables.

Regarding the symmetric tensor valued functions, we see that they are linear combinations of $(\mathcal{W} \mathcal{V}+\mathcal{V W}) \mathcal{Z}-\mathcal{Z}(\mathcal{W} \mathcal{V}+\mathcal{V} \mathcal{W})$ and of $(22)_{1-5,7-10}$. In fact, by writing $\phi^{i j}$ as a linear combination of these tensors, we have that the component 34 gives the coefficient of $(\mathcal{W} \mathcal{V}+\mathcal{V} \mathcal{W}) \mathcal{Z}-\mathcal{Z}(\mathcal{W} \mathcal{V}+\mathcal{V} \mathcal{W})$; for the other components we proceed as done after the list (22) except for the part concerning the element $(22)_{6}$. All the tensors here used are already present in the previous tables.

For the subcases which remain to consider, we can repeat what said after Table $2 T s y$ up to eq. (23). After that, from $\operatorname{tr} \mathcal{V} \mathcal{W}, \operatorname{tr} \mathcal{V}^{2} \mathcal{W}^{2}, \operatorname{tr} \mathcal{W}^{2}$ we obtain $W^{12}$, the quantity $(24)$ and $\left(W^{34}\right)^{2}$ for every tensor $\mathcal{W}$. 


\subsection{Subcase $(2,3):$ There is a skew-symmetric tensor $\mathcal{W}$ such that the quantity $(24)$ is different from zero.}

With a rotation of the axis 3 and 4 we obtain $W^{14}=0$, leaving unchanged $\mathcal{V}$ and $W^{12}$. After that, eq. $(14)_{2}$ gives $W^{23}=0$.

Moreover, from the hypothesis that the quantity (24) is not zero, and from $(14)_{1}$ we obtain $\left(W^{13}\right)^{2} \neq 0$. By eventually changing the versus of both the axis 1 and 2 we have that $W^{13}>0$, leaving unchanged all the previous results. From $\left(W^{13}\right)^{2}$ we deduce $W^{13}$. Moreover, for eq. (14), we have $\left(W^{24}\right)^{2} \neq 0$. By eventually changing the versus of the axis 4 we have that $W^{24}>0$, leaving unchanged all the previous results; from $\left(W^{24}\right)^{2}$ we deduce $W^{24}$. In particular we have $W^{24}=W^{13}$. From $t r \mathcal{V} \mathcal{W}^{3}$ we deduce the value of $W^{34}$.

For every other tensor $\mathcal{U}$, from $\operatorname{tr} \mathcal{V U}$ we obtain $U^{12}$; from $\operatorname{tr} \mathcal{V}^{2}(\mathcal{W U}+\mathcal{U} \mathcal{W})$ we obtain $W^{13} U^{13}+W^{24} U^{24}$ which, jointly with $(23)_{1}$ written with $\mathcal{U}$ instead of $\mathcal{Z}$, gives $U^{13}$ and $U^{24}$.

From $\operatorname{tr} \mathcal{V W U W}$ we obtain $U^{34}$.

From $\operatorname{tr} \mathcal{W}(\mathcal{V U}-\mathcal{U V})$ we obtain $W^{13} U^{23}-W^{24} U^{14}$ which, jointly with $(23)_{2}$ written with $\mathcal{U}$ instead of $\mathcal{Z}$, gives $U^{14}$ and $U^{23}$. We note now that the scalars here used are present in the set $S$.

Regarding the tensor valued functions, $\phi^{i j}$, we can repeat the passages of subsection $(1,3)$ from the transformation $P$ defined in eq. (25) up to the list (26), except that this last one has to be substituted by

$$
\mathcal{V}, \mathcal{W} \mathcal{W}, \mathcal{V}^{2} \mathcal{W}+\mathcal{W} \mathcal{V}^{2}, \mathcal{W} \mathcal{V}-\mathcal{V} \mathcal{W}
$$

After that we go on by writing $\phi^{i j}$ as a linear combination of these tensors; the component 34 allows to obtain the coefficient of $(38)_{2}$, the components 14 and 23 give two equivalent equations (because $\phi^{23}=-\phi^{14}$ ) which give the coefficient of $(38)_{4}$, the components 13 and 24 give two equivalent equations (because $\phi^{13}=\phi^{24}$ ) which determine the coefficient of $(38)_{3}$, the component 12 allows to obtain the coefficient of $(38)_{1}$.

We conclude that there is no need to insert a new table, because the tensors (38) are already present in the previous tables.

Regarding the symmetric tensor valued functions, we see that they are linear combinations of

$$
\mathcal{I}, \mathcal{V}^{2}, \mathcal{V}^{2} \mathcal{W}-\mathcal{W} \mathcal{V}^{2}, \mathcal{W V}+\mathcal{V W}
$$

In fact, by writing $\phi^{i j}$ as a linear combination of these tensors, we have that the components 14 and 23 give two equivalent equations (because $\phi^{14}=-\phi^{23}$ ) 
which allow to determine the coefficient of $(39)_{4}$. Similarly, the components 13 and 24 give two equivalent equations (because $\phi^{13}=\phi^{24}$ ) which allow to determine the coefficient of $(39)_{3}$.

The components 12 and 34 give identities (because $\phi^{12}=0, \phi^{34}=0$ ). The components 11, 22, 33 and 44 give four equations of which only two are independent and give the coefficients of $(39)_{1,2}$.

We conclude also here that there is no need to insert a new table, because the tensors (39) are already present in the previous tables.

\subsection{Subcase $(2,4)$ : For every skew-symmetric tensor $\mathcal{W}$ we have that the quantity $(24)$ is zero.}

It follows that $W^{13}=0, W^{14}=0, W^{23}=0, W^{24}=0$. Moreover, for every tensor $\mathcal{W}$ from $\operatorname{tr} \mathcal{V W}$ we obtain $W^{12}$ and, from $\operatorname{tr} \mathcal{W}^{2}$ we deduce $\left(W^{34}\right)^{2}$.

Regarding the representation for second order tensors $\phi^{i j}$ we note that, by changing the versus of both the axis 1 and 2, the independent variables remain unchanged so that every function depending on them must also remain unchanged. Instead of this, for the transformation law of tensors, we have that $\phi^{13}, \phi^{14}, \phi^{23}, \phi^{24}$ change sign. It follows that eq. (28) holds also in this subcase. Moreover, regarding the symmetric tensor valued functions, besides eq. (28), we have also eq. (29). In fact, with the change of reference frame (25) we have that $\phi^{11}, \phi^{22}, \phi^{12}, \phi^{33}, \phi^{44}, \phi^{34}$ transform themselves in $\phi^{22}, \phi^{11},-\phi^{12}, \phi^{44}, \phi^{33}$, $-\phi^{34}$ respectively; on the other hand, they must remain unchanged because the independent variables remain unchanged. Consequently, we can say that every symmetric tensor valued function is a linear combination of $\mathcal{I}$ and $\mathcal{V}^{2}$, which are already present in the previous Tables. For scalars and skew-symmetric tensor valued functions, we consider the following two subcases.

\subsection{Subcase $(2,4,1)$ : There is a skew-symmetric tensor $\mathcal{W}$ such that $\left(W^{34}\right)^{2} \neq 0$.}

We choose the versus of the axis 4 so that $W^{34}>0$ and determine it from $\left(W^{34}\right)^{2}$. For every other tensor $\mathcal{U}$, its only unknown components are $U^{12}$ and $U^{34}$; we determine them from $\operatorname{tr} \mathcal{V U}$ and $\operatorname{tr} \mathcal{W U}$.

Regarding the skew-symmetric tensor valued functions, thanks to eq. (28), we have that they are linear combinations of $\mathcal{V}$ and $\mathcal{W}$, which are already present in the previous Tables. 


\subsection{Subcase $(2,4,2)$ : For every skew-symmetric tensor $\mathcal{W}$ we have that $\left(W^{34}\right)^{2}=0$.}

In this subcase all the skew-symmetric tensors, among the independent variables, are proportional to $\mathcal{V}$ and have been already obtained from $\operatorname{tr} \mathcal{V W}$. Regarding the representation for second order skew-symmetric tensors $\phi^{i j}$ we note that, by changing the versus of the axis 4 the independent variables remain unchanged so that every function depending on them must also remain unchanged. Instead of this, for the transformation law of tensors, we have that $\phi^{34}$ changes sign. It follows that $\phi^{34}=0$ holds, besides eq. (28). Consequently, every skewsymmetric tensor valued function is proportional to $\mathcal{V}$. This concludes the case (2). Let us proceed with the new one.

\section{Case (3) : For every skew-symmetric tensor $\mathcal{W}$ we have that the scalar $(1)_{1}$ is zero.}

In the reference frame where $\mathcal{W}=\left(\begin{array}{cccc}0 & a & 0 & 0 \\ -a & 0 & 0 & 0 \\ 0 & 0 & 0 & b \\ 0 & 0 & -b & 0\end{array}\right)$ the condition that $(1)_{1}$ is zero becomes $4\left(a^{2}-b^{2}\right)^{2}=0$ so that $a^{2}=b^{2}$; it follows also that

$$
\mathcal{W}^{2}=-a^{2} \mathcal{I}=\left(-\frac{1}{4} \operatorname{tr} \mathcal{W}^{2}\right) \mathcal{I}
$$

Eq. (40) has been found in a particular reference frame but, for its covariant form, it holds in every other frame. Writing it in a generic frame, we have for every skew-symmetric tensor that $\left(W^{2}\right)^{11}=\left(W^{2}\right)^{22},\left(W^{2}\right)^{33}=\left(W^{2}\right)^{44}$, $\left(W^{2}\right)^{11}=\left(W^{2}\right)^{44},\left(W^{2}\right)^{i j}=0$ for $i \neq j$, or

$$
\begin{aligned}
& \left(W^{13}\right)^{2}+\left(W^{14}\right)^{2}=\left(W^{23}\right)^{2}+\left(W^{24}\right)^{2}, \\
& \left(W^{13}\right)^{2}+\left(W^{23}\right)^{2}=\left(W^{14}\right)^{2}+\left(W^{24}\right)^{2}, \\
& \left(W^{12}\right)^{2}+\left(W^{13}\right)^{2}=\left(W^{34}\right)^{2}+\left(W^{24}\right)^{2}, \\
& W^{13} W^{23}+W^{14} W^{24}=0, W^{12} W^{23}-W^{14} W^{34}=0, \\
& W^{12} W^{24}+W^{13} W^{34}=0, W^{12} W^{13}+W^{34} W^{24}=0, \\
& W^{12} W^{14}-W^{34} W^{23}=0, W^{13} W^{14}+W^{23} W^{24}=0 .
\end{aligned}
$$

These conditions can be expressed in a more elegant way as follows. Firstly, we note that there exist $\rho, \mu, \vartheta, \varphi$ with $\rho \geq 0, \mu \geq 0$, such that

$$
W^{23}=-\rho \sin \vartheta, W^{24}=\rho \cos \vartheta, W^{13}=\mu \cos \varphi, W^{14}=\mu \sin \varphi .
$$


After that, eqs. $(41)_{1,4}$ become $\mu=\rho, \rho^{2} \sin (\varphi-\vartheta)=0$; if $\rho \neq 0$, this last equation has the solution $\varphi=\vartheta+h \pi$. It follows that eqs. (42) become $W^{23}=-\rho \sin \vartheta$,

$$
W^{24}=\rho \cos \vartheta, W^{13}=(-1)^{h} \rho \cos \vartheta, W^{14}=(-1)^{h} \rho \sin \vartheta .
$$

Obviously, these relations hold also if $\rho=0$; moreover, we have $h=0$ or $h=1$ in order to have distinct expressions. By substituting eqs. (43) into eqs. (41), we find $\left(W^{12}\right)^{2}=\left(W^{34}\right)^{2}, \rho \sin \vartheta W^{12}+(-1)^{h} \rho \sin \vartheta W^{34}=0, \rho \cos \vartheta W^{12}+$ $(-1)^{h} \rho \cos \vartheta W^{34}=0$; consequently, if $\rho \neq 0$, we have

$$
W^{34}=(-1)^{h+1} W^{12} .
$$

If $\rho=0$, eqs. (43) become $W^{23}=0, W^{24}=0, W^{13}=0, W^{14}=0$ and eqs. (41) give $\left(W^{12}\right)^{2}=\left(W^{34}\right)^{2}$ so that, also in this case, eq. (44) holds. In other words, eqs. (41) are equivalent to (43) and (44).

We conclude that every skew-symmetric tensor $\mathcal{W}$ is determined by the four quantities $W^{12}, \rho, \vartheta$ and $h$; moreover, this last one may assume only the values $h=0$ and $h=1$. In the sequel, the following scalar will be used,

$$
4 t r \mathcal{V W} \mathcal{V W}+\left(\operatorname{tr} \mathcal{W}^{2}\right)\left(\operatorname{tr} \mathcal{V}^{2}\right)-2(\operatorname{tr} \mathcal{V} \mathcal{W})^{2}
$$

\subsection{Subcase $(3,1)$ : There are two skew-symmetric tensors $\mathcal{V}$ and $\mathcal{W}$ such that the scalar $(45)$ is not zero.}

Obviously, the tensor $\mathcal{V}$ cannot be zero.We choose the reference frame where $\mathcal{V}=\left(\begin{array}{cccc}0 & a & 0 & 0 \\ -a & 0 & 0 & 0 \\ 0 & 0 & 0 & a \\ 0 & 0 & -a & 0\end{array}\right)$ with $a>0$

With the same procedure used after eq. (5) up to the first line after eq. (8), we can find a reference frame where $W^{14}=0, W^{23}=0$.

After that, the condition that (45) is not zero becomes $8 a^{2}\left[\left(W^{12}-W^{34}\right)^{2}+\right.$ $\left.\left(W^{13}+W^{24}\right)^{2}\right] \neq 0$. This condition, thanks to eqs. (43) and (44), becomes $8 a^{2}\left[\left(W^{12}\right)^{2}+\rho^{2} \cos ^{2} \vartheta\right]\left[1+(-1)^{h}\right] \neq 0$; from this condition it follows that $h$ cannot be 1 , so that we must have $h=0$; moreover, $W^{12}$ and $W^{13}$ cannot be both zero.

From (43) and (44) we deduce that $W^{13}=W^{24}, W^{34}=-W^{12}$.

Now we consider the transformation

$$
P=\left(\begin{array}{cccc}
\cos \theta & 0 & 0 & \sin \theta \\
0 & \cos \theta & -\sin \theta & 0 \\
0 & \sin \theta & \cos \theta & 0 \\
-\sin \theta & 0 & 0 & \cos \theta
\end{array}\right) \text { where } \theta \text { is a solution of the equation }
$$


$W^{13} \cos (2 \theta)-W^{12} \sin (2 \theta)=0$; by using it, the tensor

$$
\left(\begin{array}{cccc}
0 & W^{12} & W^{13} & 0 \\
-W^{12} & 0 & 0 & W^{13} \\
-W^{13} & 0 & 0 & -W^{12} \\
0 & -W^{13} & W^{12} & 0
\end{array}\right)
$$

transforms itself in

$$
\left(\begin{array}{cccc}
0 & \left(W^{\prime}\right)^{12} & 0 & 0 \\
-\left(W^{\prime}\right)^{12} & 0 & 0 & 0 \\
0 & 0 & 0 & -\left(W^{\prime}\right)^{12} \\
0 & 0 & \left(W^{\prime}\right)^{12} & 0
\end{array}\right)
$$

and the tensor $\mathcal{V}$ remains unchanged.

In other words, a reference frame exists where $W^{13}=0, W^{14}=0, W^{23}=0$, $W^{24}=0, W^{34}=-W^{12}$.

After that, we note that the transformation $P=\left(\begin{array}{llll}0 & 0 & 1 & 0 \\ 0 & 0 & 0 & 1 \\ 1 & 0 & 0 & 0 \\ 0 & 1 & 0 & 0\end{array}\right)$ acts on

$\left(\begin{array}{cccc}0 & W^{12} & 0 & 0 \\ -W^{12} & 0 & 0 & 0 \\ 0 & 0 & 0 & W^{34} \\ 0 & 0 & -W^{34} & 0\end{array}\right)$ simply exchanging $W^{12}$ and $W^{34}$. Consequently, it leaves $\mathcal{V}$ unchanged and allows to find a reference frame where $W^{12}>0$.

After that, from $\operatorname{tr} \mathcal{V}^{2}$ we find $a$ and, from $\operatorname{tr} \mathcal{W}^{2}$ we find $W^{12}$ and, consequently, the whole tensor $\mathcal{W}$.

For every other tensor $\mathcal{Z}$, from $\operatorname{tr} \mathcal{V} \mathcal{Z}$ and $\operatorname{tr} \mathcal{W} \mathcal{Z}$ we find $Z^{12}$ and $Z^{34}$; after that, from $\operatorname{tr} \mathcal{Z}^{2}$ we find $\left(Z^{13}\right)^{2}+\left(Z^{14}\right)^{2}+\left(Z^{23}\right)^{2}+\left(Z^{24}\right)^{2}$.

For the sequel, the following scalar will be useful

$$
\begin{aligned}
& 2 \operatorname{tr} \mathcal{I}_{1}(\mathcal{Z} \mathcal{T}+\mathcal{T} \mathcal{Z}) \mathcal{I}_{1}(\mathcal{Z} \mathcal{T}+\mathcal{T} \mathcal{Z})-\left[\operatorname{tr} \mathcal{I}_{1}(\mathcal{Z} \mathcal{T}+\mathcal{T} \mathcal{Z})\right]^{2} \\
& \text { where } \mathcal{I}_{1}=\frac{1}{a\left(W^{34}-W^{12}\right)}\left(a W^{34} \mathcal{I}+\mathcal{V W}\right)
\end{aligned}
$$

From the second of these equations, it follows that $\mathcal{I}_{1}=\operatorname{diag}(1,1,0,0)$. 


\subsection{Subcase $(3,1,1)$ : There are two skew-symmetric tensors $\mathcal{Z}$ and $\mathcal{T}$ such that the scalar (46) is different from zero.}

We note firstly that the scalar present in (46) can be obtained from

$$
\begin{aligned}
& \operatorname{tr}(\mathcal{V W}+\mathcal{W} \mathcal{V})(\mathcal{Z} \mathcal{T}+\mathcal{T} \mathcal{Z})(\mathcal{V W}+\mathcal{W} \mathcal{V})(\mathcal{Z} \mathcal{T}+\mathcal{T} \mathcal{Z}) \\
& \operatorname{tr}(\mathcal{V W}+\mathcal{W} \mathcal{V})(\mathcal{Z} \mathcal{T} \mathcal{Z}+\mathcal{T} \mathcal{Z} \mathcal{T}), \operatorname{tr} \mathcal{Z} \mathcal{T} \mathcal{T}, \operatorname{tr} \mathcal{W}^{2}, \\
& \operatorname{tr}(\mathcal{V W}+\mathcal{W} \mathcal{V})(\mathcal{Z} \mathcal{T}+\mathcal{T} \mathcal{Z}), \operatorname{tr}(\mathcal{Z} \mathcal{T}+\mathcal{T} \mathcal{Z})=2 \operatorname{tr} \mathcal{Z} \mathcal{T}, \operatorname{tr} \mathcal{T}^{2}
\end{aligned}
$$

where we have used the property that $\mathcal{T}^{2}$ and $\mathcal{Z}^{2}$ are proportional to the identity matrix. Now the tensor $\mathcal{I}_{1}(\mathcal{Z} \mathcal{T}+\mathcal{T} \mathcal{Z}) \mathcal{I}_{1}$ is symmetric and only its components $11,12,21,22$ may be different from zero; consequently, with a suitable rotation of the axis 1 and 2, we obtain that its new component 12 is zero and, moreover, $\left[\mathcal{I}_{1}(\mathcal{Z} \mathcal{T}+\mathcal{T} \mathcal{Z}) \mathcal{I}_{1}\right]^{11} \leq\left[\mathcal{I}_{1}(\mathcal{Z} \mathcal{T}+\mathcal{T} \mathcal{Z}) \mathcal{I}_{1}\right]^{22}$. After that, the condition that (46) is not zero, now becomes $\left[\mathcal{I}_{1}(\mathcal{Z} \mathcal{T}+\mathcal{T} \mathcal{Z}) \mathcal{I}_{1}\right]^{11} \neq\left[\mathcal{I}_{1}(\mathcal{Z} \mathcal{T}+\mathcal{T} \mathcal{Z}) \mathcal{I}_{1}\right]^{22}$, so that we now have

$$
\left[\mathcal{I}_{1}(\mathcal{Z} \mathcal{T}+\mathcal{T} \mathcal{Z}) \mathcal{I}_{1}\right]^{11}<\left[\mathcal{I}_{1}(\mathcal{Z} \mathcal{T}+\mathcal{T} \mathcal{Z}) \mathcal{I}_{1}\right]^{22}
$$

This is equivalent to $Z^{2 a} T^{a 2}>Z^{1 a} T^{a 1}$ (where the summation convention over the repeated index $a$ is used), or

$$
Z^{23} T^{32}+Z^{24} T^{42}>Z^{13} T^{31}+Z^{14} T^{41}
$$

But, from (43) we have

$$
\begin{aligned}
& Z^{23}=-\rho_{Z} \sin \vartheta_{Z}, Z^{24}=\rho_{Z} \cos \vartheta_{Z}, Z^{13}=(-1)^{h_{Z}} \rho_{Z} \cos \vartheta_{Z}, \\
& Z^{14}=(-1)^{h_{Z}} \rho_{Z} \sin \vartheta_{Z}, T^{23}=-\rho_{T} \sin \vartheta_{T}, \\
& T^{24}=\rho_{T} \cos \vartheta_{T}, T^{13}=(-1)^{h_{T}} \rho_{T} \cos \vartheta_{T}, T^{14}=(-1)^{h_{T}} \rho_{T} \sin \vartheta_{T} .
\end{aligned}
$$

Thanks to these expressions, eq. (48) now becomes

$$
\rho_{Z} \rho_{T}\left[(-1)^{h_{Z}+h_{T}}-1\right] \cos \left(\vartheta_{T}-\vartheta_{Z}\right)>0 .
$$

Consequently, $h_{Z}$ and $h_{T}$ cannot be both zero, nor both 1 ; one of them will be zero and the other 1 . We know what among them is zero and what is 1 because, from $\operatorname{tr} \mathcal{V} \mathcal{Z} \mathcal{Z}$ we obtain $Z^{13} Z^{24}-Z^{23} Z^{14}$ which, thanks to eq. (49), can be expressed as $(-1)^{h_{Z}} \rho_{Z}^{2}$. Similarly, from $\operatorname{tr} \mathcal{V} \mathcal{T} \mathcal{V} \mathcal{T}$ we obtain $(-1)^{h_{T}} \rho_{T}^{2}$. Except for exchanging $\mathcal{Z}$ and $\mathcal{T}$, we may assume that $h_{Z}=0$ and $h_{T}=1$ (This does not effect the results because in every element of the tables involving $\mathcal{Z}$ and $\mathcal{T}$ it is understood that the same element with $\mathcal{Z}$ and $\mathcal{T}$ exchanged has to 
be considered).

After that, eq. (50) becomes

$$
\rho_{Z} \neq 0, \rho_{T} \neq 0, \cos \left(\vartheta_{T}-\vartheta_{Z}\right)<0 .
$$

Let us consider now $P=\left(\begin{array}{cccc}1 & 0 & 0 & 0 \\ 0 & 1 & 0 & 0 \\ 0 & 0 & \cos \theta & \sin \theta \\ 0 & 0 & -\sin \theta & \cos \theta\end{array}\right)$ that is a rotation of the axis 3 and 4 . We note that $P^{T} \mathcal{Z} P$ leaves unchanged $Z^{12}, Z^{34}, \rho_{Z}, h_{Z}$ but transforms $\theta_{Z}$ in $\theta_{Z}+\theta$; similarly, $P^{T} \mathcal{T} P$ leaves unchanged $T^{12}, T^{34}, \rho_{T}, h_{T}$ but transforms $\theta_{T}$ in $\theta_{T}+\theta$.

By choosing $\theta=-\theta_{T}$, we have that the new components $Z^{14}$ and $Z^{23}$ are zero, and in the new reference frame we have also $Z^{13}=Z^{24}=\rho_{Z}>0$, because $h_{Z}=0$. While, from $h_{T}=1$, it follows $T^{24}=-T^{13}, T^{23}=T^{14}$; moreover, $T^{\prime 13}=-\rho_{T} \cos \left(\vartheta_{T}-\vartheta_{Z}\right)>0$, thanks to the inequality (51).

Moreover, the above mentioned rotation leaves unchanged $\mathcal{I}_{1}(\mathcal{Z} \mathcal{T}+\mathcal{T Z}) \mathcal{I}_{1}$. This fact allows to use the condition $\left[\mathcal{I}_{1}(\mathcal{Z} \mathcal{T}+\mathcal{T} \mathcal{Z}) \mathcal{I}_{1}\right]^{12}=0$, that is, $-Z^{13} T^{23}-$ $T^{14} Z^{24}=0$ which, for the condition $Z^{13}=Z^{24}>0$, becomes $T^{23}+T^{14}=0$ which, jointly with $T^{23}=T^{14}$, becomes $T^{23}=0, T^{14}=0$.

A this point, the components of $\mathcal{Z}$ and $\mathcal{T}$ are known. In fact, from $\operatorname{tr} \mathcal{Z}^{2}$ we find $\left(Z^{13}\right)^{2}$ from which $Z^{13}$, because $Z^{13}>0$; similarly, from $\operatorname{tr} \mathcal{T}^{2}$ we find $T^{13}$. For every other tensor $\mathcal{U}$, we find $U^{12}$ and $U^{34}$ from $\operatorname{tr} \mathcal{V U}$ and $\operatorname{tr} \mathcal{W U}, U^{13}$ and $U^{24}$ from $\operatorname{tr} \mathcal{Z U}$ and $\operatorname{tr} \mathcal{T U}, U^{23}-U^{14}$ and $U^{23}+U^{14}$ from $\operatorname{tr} \mathcal{Z}(\mathcal{W U}-\mathcal{U W})$ and $\operatorname{tr} \mathcal{T}(\mathcal{V U}-\mathcal{U V})$ respectively. The new scalars used after eq. (46) are present in the set $S$.

Regarding the skew-symmetric tensor valued functions, we see that they are linear combinations of

$$
\mathcal{V}, \mathcal{W}, \mathcal{Z}, \mathcal{T}, \mathcal{Z W}-\mathcal{W Z}, \mathcal{T} \mathcal{V}-\mathcal{V} \mathcal{T}
$$

In fact, by writing $\phi^{i j}$ as a linear combination of these tensors, we have that $\phi^{14}+\phi^{23}$ gives the coefficient of $(52)_{6}$, $\phi^{14}-\phi^{23}$ gives the coefficient of $(52)_{5}$, $\phi^{13}-\phi^{24}$ gives the coefficient of $(52)_{4}$, $\phi^{13}+\phi^{24}$ gives the coefficient of $(52)_{3}$, $\phi^{12}-\phi^{34}$ gives the coefficient of $(52)_{2}$, $\phi^{12}+\phi^{34}$ gives the coefficient of $(52)_{1}$.

In this way we have not used new elements to insert in new tables.

Regarding the symmetric tensor valued functions, we see that they are linear 
combinations of

$$
\begin{aligned}
& \mathcal{I}_{1}, \mathcal{I}_{2}, \mathcal{I}_{1}(\mathcal{Z} \mathcal{T}+\mathcal{T} \mathcal{Z}) \mathcal{I}_{1}, \mathcal{I}_{2}(\mathcal{Z} \mathcal{T}+\mathcal{T} \mathcal{Z}) \mathcal{I}_{2} \\
& \mathcal{I}_{1}(\mathcal{Z} \mathcal{T}+\mathcal{T} \mathcal{Z}) \mathcal{I}_{1} \mathcal{V}-\mathcal{V} \mathcal{I}_{1}(\mathcal{T} \mathcal{Z}+\mathcal{Z} \mathcal{T}) \mathcal{I}_{1} \\
& \mathcal{I}_{2}(\mathcal{Z} \mathcal{T}+\mathcal{T} \mathcal{Z}) \mathcal{I}_{2} \mathcal{W}-\mathcal{W} \mathcal{I}_{2}(\mathcal{T} \mathcal{Z}+\mathcal{Z} \mathcal{T}) \mathcal{I}_{2}, \mathcal{I}_{1} \mathcal{Z}-\mathcal{Z} \mathcal{I}_{1} \\
& \mathcal{I}_{1} \mathcal{T}-\mathcal{T I}_{1}, \mathcal{I}_{1} \mathcal{Z} \mathcal{W}+\mathcal{W} \mathcal{Z} \mathcal{I}_{1}, \mathcal{I}_{1} \mathcal{T} \mathcal{V}+\mathcal{V} \mathcal{T} \mathcal{I}_{1} \\
& \text { where } \quad \mathcal{I}_{2}=\mathcal{I}-\mathcal{I}_{1}=\operatorname{diag}(0,0,1,1)
\end{aligned}
$$

In fact, by writing $\phi^{i j}$ as a linear combination of these tensors, we have that $\phi^{34}$ gives the coefficient of $(53)_{6}$,

$\phi^{14}+\phi^{23}$ gives the coefficient of $(53)_{10}$,

$\phi^{14}-\phi^{23}$ gives the coefficient of $(53)_{9}$,

$\phi^{13}-\phi^{24}$ gives the coefficient of $(53)_{8}$,

$\phi^{13}+\phi^{24}$ gives the coefficient of $(53)_{7}$,

$\phi^{12}$ gives the coefficient of $(53)_{4}$,

$\phi^{11}$ and $\phi^{22}$ give the coefficients of $(53)_{1,3}$,

$\phi^{33}$ and $\phi^{44}$ give the coefficients of $(53)_{2,4}$.

We insert in the next table the new tensors used.

The table $4 T s y$

For every set of four tensors: $\mathcal{V W}(\mathcal{Z} \mathcal{T}+\mathcal{T} \mathcal{Z}) \mathcal{W} \mathcal{V}, \mathcal{V W}(\mathcal{Z} \mathcal{T}+\mathcal{T} \mathcal{Z})+(\mathcal{Z} \mathcal{T}+$ $\mathcal{T} \mathcal{Z}) \mathcal{W} \mathcal{V}$

$\mathcal{V W}(\mathcal{Z} \mathcal{T}+\mathcal{T} \mathcal{Z}) \mathcal{W}-\mathcal{W}(\mathcal{Z} \mathcal{T}+\mathcal{T} \mathcal{Z}) \mathcal{W} \mathcal{V}, \mathcal{W} \mathcal{Z} \mathcal{W}+\mathcal{W} \mathcal{Z} \mathcal{W}$

where we have used the symmetry of $\mathcal{V W}$. In the next subcase, the following quantity will be used,

$$
\left(Z^{13}\right)^{2}+\left(Z^{14}\right)^{2}+\left(Z^{23}\right)^{2}+\left(Z^{24}\right)^{2}
$$

\subsection{Subcase $(3,1,2)$ : However we take two skew-symmetric tensors $\mathcal{Z}$ and $\mathcal{T}$ we have that the scalar (46) is zero, but there is one of them $\mathcal{Z}$ such that the quantity (54) is different from zero.}

Through a rotation of the axis 3 and 4 we obtain $Z^{14}=0$. After that, eq. $(43)_{1,4}$ with $\mathcal{Z}$ instead of $\mathcal{W}$ give $Z^{23}=0$. From $(41)_{1}$ we have $\left(Z^{13}\right)^{2}=\left(Z^{24}\right)^{2}$. This allows to obtain $\left(Z^{24}\right)^{2}$ from $\operatorname{tr} \mathcal{Z}^{2}$. By eventually changing the versus of both axis 1 and 2, we obtain $Z^{24}>0$ and deduce its value from $\left(Z^{24}\right)^{2}$.

After that, $\operatorname{tr} \mathcal{V} \mathcal{Z} \mathcal{V}$ gives $Z^{13}$. In other words, from (43) with $\mathcal{Z}$ instead of $\mathcal{W}$ we have found $\vartheta=0$ and we know if $h=0$ (that is $W^{13}>0$ ) or if $h=1$ (that is $W^{13}<0$ ). 
After that, the fact that the scalar (46) is zero says that $(\mathcal{Z T}+\mathcal{T} \mathcal{Z})^{12}=0,(\mathcal{Z} \mathcal{T}+\mathcal{T} \mathcal{Z})^{11}=(\mathcal{Z} \mathcal{T}+\mathcal{T} \mathcal{Z})^{22}$ for every $\mathcal{T}$, that is $\rho_{Z} \rho_{T}\left[(-1)^{h_{Z}}-(-1)^{h_{T}}\right] \sin \vartheta_{T}=0, \rho_{Z} \rho_{T}\left[1-(-1)^{h_{Z}+h_{T}}\right] \cos \vartheta_{T}=0$, from which $\rho_{T}=0$ or $h_{Z}+h_{T}$ is an even number; in the second of these cases we have that

- If $Z^{13}=Z^{24}$, then $T^{13}=T^{24}, T^{23}=-T^{14} \forall \mathcal{T}$,

- If $Z^{13}=-Z^{24}$, then $T^{13}=-T^{24}, T^{23}=T^{14} \forall \mathcal{T}$.

Obviously, this result holds also in the case $\rho_{T}=0$, that is, $T^{13}=0, T^{14}=0$, $T^{23}=0, T^{24}=0$. After that, $\operatorname{tr} \mathcal{V} \mathcal{T}$ and $\operatorname{tr} \mathcal{W} \mathcal{T}$ give us $T^{12}$ and $T^{34} ; \operatorname{tr} \mathcal{Z} \mathcal{T}$ gives $Z^{13} T^{13}+Z^{24} T^{24}$ from which $T^{24}$ and, obviously, $T^{13}$.

Finally, if $Z^{13}=Z^{24}$, we obtain $T^{14}$ (and consequently, also $T^{23}$ ) from $\operatorname{tr} \mathcal{W}(\mathcal{Z} \mathcal{T}$ $\mathcal{T Z})$, while

if $Z^{13}=-Z^{24}$, we obtain $T^{14}$ (and consequently, also $T^{23}$ ) from $\operatorname{tr} \mathcal{V}(\mathcal{Z} \mathcal{T}-\mathcal{T} \mathcal{Z}$ ). In this way we have found whatever tensor $\mathcal{T}$.

For what concerns the tensorial functions, let us firstly consider the sets

$$
\begin{gathered}
\mathcal{V}, \mathcal{W}, \mathcal{Z}, \mathcal{Z} \mathcal{W}-\mathcal{W Z} \\
\mathcal{I}, \mathcal{W} \mathcal{V}+\mathcal{V} \mathcal{W},(\mathcal{V W}+\mathcal{W} \mathcal{V}) \mathcal{Z}-\mathcal{Z}(\mathcal{V W}+\mathcal{W} \mathcal{V}), \mathcal{Z} \mathcal{V}+\mathcal{V} \mathcal{Z} \\
\mathcal{V}, \mathcal{W}, \mathcal{Z}, \mathcal{Z} \mathcal{V}-\mathcal{V} \mathcal{Z} \\
\mathcal{I}, \mathcal{W} \mathcal{V}+\mathcal{V} \mathcal{W},(\mathcal{V W}+\mathcal{W} \mathcal{V}) \mathcal{Z}-\mathcal{Z}(\mathcal{V W}+\mathcal{W} \mathcal{V}), \mathcal{Z} \mathcal{W}+\mathcal{W} \mathcal{Z}
\end{gathered}
$$

After that, we may repeat the considerations above written starting from the line before eq. (25) up to the end of the subcase $(1,3)$, but substituting $(\mathcal{W}, \mathcal{Z})$ with $(\mathcal{Z}, \mathcal{T})$; moreover, in the case $W^{24}=W^{13}$ we have to substitute eq. (26) with (55) and eq. (27) with (56), while in the case $W^{24}=-W^{13}$ we have to substitute eq. (26) with (57) and eq. (27) with (58).

\subsection{Subcase $(3,1,3)$ : However we take two skew-symmetric tensors $\mathcal{Z}$ and $\mathcal{T}$ we have that the scalar (46) is zero, and the quantity} (54) is zero.

It follows that $Z^{14}=0, Z^{23}=0, Z^{13}=0, Z^{24}=0$. Moreover, every tensor $\mathcal{Z}$ can be obtained from $\operatorname{tr} \mathcal{V} \mathcal{Z}$ and $\operatorname{tr} \mathcal{W} \mathcal{Z}$ which are scalars already present in the set $S$. 
Regarding the representation for second order tensors $\phi^{i j}$ we note that, by changing the versus of both the axis 1 and 2, the independent variables remain unchanged so that every function depending on them must also remain unchanged. Instead of this, for the transformation law of tensors, we have that $\phi^{13}, \phi^{14}, \phi^{23}, \phi^{24}$ transform themselves into $-\phi^{13},-\phi^{14},-\phi^{23},-\phi^{34}$ respectively. It follows that $\phi^{13}=0, \phi^{14}=0, \phi^{23}=0, \phi^{24}=0$. It follows that every skew-symmetric tensor valued function is a linear combination of $\mathcal{V}$ and $\mathcal{W}$.

Regarding the symmetric tensor valued functions, besides $\phi^{13}=0, \phi^{14}=0$, $\phi^{23}=0, \phi^{24}=0$, we have also $\phi^{11}=\phi^{22}, \phi^{12}=0, \phi^{33}=\phi^{44}, \phi^{34}=0$. In fact, with the change of reference frame (25) we have that $\phi^{11}, \phi^{22}, \phi^{12}, \phi^{33}, \phi^{44}$, $\phi^{34}$ transform themselves in $\phi^{22}, \phi^{11},-\phi^{12}, \phi^{44}, \phi^{33},-\phi^{34}$ respectively; on the other hand, they must remain unchanged because the independent variables remain unchanged.

After that, we find that every symmetric tensor valued function is a linear combination of $\mathcal{I}$ and $\mathcal{V} \mathcal{W}+\mathcal{W} \mathcal{V}$, which are already present in previous tables. Let us proceed with a new subcase. It is described in terms of the quantities

$$
(\operatorname{tr} \mathcal{W} \mathcal{V})^{2}-\left(t r \mathcal{W}^{2}\right)\left(t r \mathcal{V}^{2}\right)
$$

\subsection{Subcase $(3,2)$ : However we take two skew-symmetric tensors $\mathcal{V}$ and $\mathcal{W}$ we have that the scalar $(45)$ is zero, but there exist two of them such that the scalar (59) is different from zero.}

From the condition that the scalar (59) is different from zero it follows $\mathcal{V} \neq 0$; consequently, there is a reference frame where

$$
\mathcal{V}=\left(\begin{array}{cccc}
0 & a & 0 & 0 \\
-a & 0 & 0 & 0 \\
0 & 0 & 0 & a \\
0 & 0 & -a & 0
\end{array}\right) \quad \text { with } a>0
$$

Through a suitable rotation of the axis 3 and 4 , we obtain $W^{14}=0$. After that, from $(43)_{1,4}$ it follows $W^{23}=0$.

The hypothesis that the scalar $(45)$ is zero becomes $8 a^{2}\left[\left(W^{12}-W^{34}\right)^{2}+\left(W^{13}+\right.\right.$ $\left.\left.W^{24}\right)^{2}\right]=0$, that is $W^{12}=W^{34}, W^{13}=-W^{24}$.

The hypothesis that the scalar $(59)$ is different from zero implies $\left(W^{13}\right)^{2}+$ $\left(W^{24}\right)^{2} \neq 0$, that is $\left(W^{13}\right)^{2} \neq 0$.

Except for an eventual inversion of both axis 1 and 2, we have $W^{13}>0$ and deduce its value from $\left(W^{13}\right)^{2}$.

After that, also $W^{24}$ is known, that is the whole tensor $\mathcal{W}$ (because its components $W^{12}=W^{34}$ can be deduced from $\left.\operatorname{tr} \mathcal{V} \mathcal{W}\right)$. 
It follows also that

$\mathcal{V} \mathcal{W}=\frac{1}{4}(\operatorname{tr} \mathcal{W}) \mathcal{I}+a\left(\begin{array}{cccc}0 & 0 & 0 & -W^{13} \\ 0 & 0 & -W^{13} & 0 \\ 0 & W^{13} & 0 & 0 \\ W^{13} & 0 & 0 & 0\end{array}\right)$.

For every other tensor $\mathcal{U}$, the hypothesis that the scalar (45) is zero says that $U^{12}=U^{34}$

$U^{24}=-U^{13}, U^{23}=U^{14}$.

From $\operatorname{tr} \mathcal{V U}$ we obtain $U^{12}$, from $\operatorname{tr} \mathcal{W U}$ we obtain $U^{13}$, from $\operatorname{tr} \mathcal{V}(\mathcal{W U}-\mathcal{U} \mathcal{W})$ we obtain $U^{14}$; in this way the whole tensor $\mathcal{U}$ has been found and we have not used new scalars.

After that, for every tensor $\phi^{i j}$, we note that through the transformation

$$
\begin{aligned}
P & =\left(\begin{array}{cccc}
0 & 1 & 0 & 0 \\
-1 & 0 & 0 & 0 \\
0 & 0 & 0 & -1 \\
0 & 0 & 1 & 0
\end{array}\right) \text { it transforms itself according to the law } \\
& \left(\begin{array}{cccc}
\phi^{11} & \phi^{12} & \phi^{13} & \phi^{14} \\
\phi^{21} & \phi^{22} & \phi^{23} & \phi^{24} \\
\phi^{31} & \phi^{32} & \phi^{33} & \phi^{34} \\
\phi^{41} & \phi^{42} & \phi^{43} & \phi^{44}
\end{array}\right) \rightarrow\left(\begin{array}{cccc}
\phi^{22} & -\phi^{21} & -\phi^{24} & \phi^{23} \\
-\phi^{12} & \phi^{11} & \phi^{14} & -\phi^{13} \\
-\phi^{42} & \phi^{41} & \phi^{44} & -\phi^{43} \\
\phi^{32} & -\phi^{31} & -\phi^{34} & \phi^{33}
\end{array}\right)
\end{aligned}
$$

whose left hand side is $\phi$ and whose right hand side is $P^{T} \phi P$ (We note that $P$ is the rotation of the axis 1 and 2 with an angle $\frac{\pi}{2}$, and the rotation of the axis 3 and 4 with an angle $-\frac{\pi}{2}$ ). The independent variables transform themselves like $\phi$; this allows us to see that they remain unchanged trough this transformation. Therefore, also $\phi$ must remain unchanged because it is a function of those variables. Consequently, if $\phi$ is symmetric, we have $\phi^{11}=\phi^{22}, \phi^{12}=0, \phi^{33}=$ $\phi^{44}, \phi^{34}=0, \phi^{13}=-\phi^{24}, \phi^{14}=\phi^{23}$; while if $\phi$ is skew-symmetric, we have $\phi^{13}=-\phi^{24}, \phi^{14}=\phi^{23}$.

We find further properties by considering the transformation

$$
P=\left(\begin{array}{cccc}
0 & 0 & 1 & 0 \\
0 & 0 & 0 & 1 \\
-1 & 0 & 0 & 0 \\
0 & -1 & 0 & 0
\end{array}\right)
$$


it transforms $\phi^{i j}$ in the following way

$$
\left(\begin{array}{llll}
\phi^{11} & \phi^{12} & \phi^{13} & \phi^{14} \\
\phi^{21} & \phi^{22} & \phi^{23} & \phi^{24} \\
\phi^{31} & \phi^{32} & \phi^{33} & \phi^{34} \\
\phi^{41} & \phi^{42} & \phi^{43} & \phi^{44}
\end{array}\right) \rightarrow\left(\begin{array}{cccc}
\phi^{33} & \phi^{34} & -\phi^{31} & -\phi^{32} \\
\phi^{43} & \phi^{44} & -\phi^{41} & -\phi^{42} \\
-\phi^{13} & -\phi^{14} & \phi^{11} & \phi^{12} \\
-\phi^{23} & -\phi^{24} & \phi^{21} & \phi^{22}
\end{array}\right)
$$

(whose left hand side is $\phi$ and whose right hand side is $P^{T} \phi P$ ) and we see that the independent variables remain unchanged trough this transformation. Therefore, also $\phi$ must remain unchanged because it is a function of those variables. Consequently, we have $\phi^{12}=\phi^{34}$; moreover, if $\phi$ is symmetric, we have also $\phi^{13}=0, \phi^{24}=0, \phi^{11}=\phi^{33}, \phi^{14}=-\phi^{23}$. This last equation, jointly with the previously known condition $\phi^{14}=\phi^{23}$ implies $\phi^{14}=0, \phi^{23}=0$; consequently, every symmetric tensorial function is proportional to the identity tensor and there is no new table to insert.

Regarding an arbitrary skew-symmetric tensorial function, we see that it is a linear combination of

$$
\mathcal{V}, \mathcal{W}, \mathcal{W} \mathcal{V}-\mathcal{V} \mathcal{~}
$$

In fact, the components 14 and 23 give two equivalent equations from which the coefficient of $(63)_{3}$ can be determined, the components 13 and 24 give two equivalent equations from which the coefficient of $(63)_{2}$ can be determined, the components 12 and 34 give two equivalent equations from which the coefficient of $(63)_{1}$ can be determined. Also here, there is no new table to insert.

\subsection{Subcase $(3,3)$ : However we take two skew-symmetric tensors $\mathcal{V}$ and $\mathcal{W}$ we have that the scalars (45) and (59) are zero, but there is one of them with $t r \mathcal{V}^{2} \neq 0$.}

Let us consider a reference frame where (60) holds. The hypothesis that the scalar $(45)$ is zero becomes $8 a^{2}\left[\left(W^{12}-W^{34}\right)^{2}+\left(W^{13}+W^{24}\right)^{2}+\left(W^{23}-W^{14}\right)^{2}\right]=$ 0 , that is $W^{12}=W^{34}, W^{13}=-W^{24}, W^{23}=W^{14}$.

After that, the hypothesis that the scalar (59) is zero becomes $-8 a^{2}\left[\left(W^{13}\right)^{2}+\left(W^{14}\right)^{2}+\left(W^{23}\right)^{2}+\left(W^{24}\right)^{2}\right]=0$, that is $W^{13}=0, W^{14}=0$, $W^{23}=0, W^{24}=0$.

After that, from $\operatorname{tr} \mathcal{V W}$ we obtain $W^{12}$, that is the whole tensor $\mathcal{W}$.

Regarding the tensorial functions $\phi^{i j}$, we see that changing the versus of both axis 1 and 2, the independent variables remain unchanged, while $\phi^{13}, \phi^{14}, \phi^{23}$, $\phi^{24}$ change sign. It follows that $\phi^{13}=0, \phi^{14}=0, \phi^{23}=0, \phi^{24}=0$. 
Moreover, with the transformation (25) the independent variables remain unchanged, while $\phi^{11}, \phi^{22}, \phi^{12}, \phi^{21}, \phi^{33}, \phi^{44}, \phi^{34}, \phi^{43}$ transform themselves in $\phi^{22}, \phi^{11},-\phi^{21},-\phi^{12}, \phi^{44}, \phi^{33},-\phi^{43},-\phi^{34}$ respectively. Consequently, if $\phi^{i j}$ is symmetric, we have $\phi^{11}=\phi^{22}, \phi^{12}=0, \phi^{33}=\phi^{44}, \phi^{34}=0$.

We find further properties by considering the transformation (61) and its consequence (62) and we see that the independent variables remain unchanged trough this transformation. Therefore, also $\phi$ must remain unchanged because it is a function of those variables. It follows that $\phi^{12}=\phi^{34}, \phi^{11}=\phi^{33}$.

Consequently, every symmetric tensorial function is proportional to the identity tensor and every arbitrary skew-symmetric tensorial function is proportional to $\mathcal{V}$.

\subsection{Subcase $(3,4)$ : For every skew-symmetric tensor $\mathcal{V}$ we have $\operatorname{tr} \mathcal{V}^{2}=0$.}

In this case we have $\mathcal{V}=0 \forall \mathcal{V}$ and we need no scalar for our corresponding representation. Regarding that for second order tensorial functions $\phi^{i j}$ we see that, by changing the versus of the first axis, the independent variables remain unchanged while $\phi^{12}, \phi^{13}, \phi^{14}$ change sign. It follows that $\phi^{12}=0, \phi^{13}=0$, $\phi^{14}=0$.

By performing the same passages with the versus of the second axis, we obtain that $\phi^{21}=0, \phi^{23}=0, \phi^{24}=0$. With a change of the versus of the third axis, we obtain $\phi^{31}=0, \phi^{32}=0, \phi^{34}=0$.

Consequently, if $\phi^{i j}$ is skew-symmetric, we have $\phi^{i j}=0$ and there is no element to insert in the corresponding representation.

Instead of this, if $\phi^{i j}$ is symmetric, we have $\phi^{i j}=\operatorname{diag}\left(\phi^{11}, \phi^{22}, \phi^{33}, \phi^{44}\right)$.

But the transformation (25) and its consequence imply that $\phi^{11}=\phi^{22}, \phi^{33}=$ $\phi^{44}$. Similarly, with the transformation

$P=\left(\begin{array}{cccc}0 & 0 & 0 & 1 \\ 0 & 0 & 1 & 0 \\ 0 & -1 & 0 & 0 \\ -1 & 0 & 0 & 0\end{array}\right)$ we obtain $\phi^{11}=\phi^{44}, \phi^{22}=\phi^{33}$.

Consequently, $\phi$ is proportional to the identity tensor.

This concludes all the possible existing cases.

We finish this article emphasizing the many possible physical applications of the Representation Theorems, such us in the framework of Extended Thermodynamics (see refs. [11]-[30]) and others fields of application. 


\section{A. Appendix: Proofs for Sect. 2}

Proof of Property 1. Let $\vec{w}$ be an eigenvector of $\mathcal{V}$ corresponding to the eigenvalue $\lambda$ and $\vec{w}^{*}$ its complex conjugate; let also $\lambda^{*}$ be the complex conjugate of $\lambda$. By multiplying $\mathcal{V} \vec{w}=\lambda \vec{w}$ on the left, times $\vec{w}^{* T}$ (that is, the transpose of $\left.\vec{w}^{*}\right)$, we obtain $\vec{w}^{* T} \mathcal{V} \vec{w}=\lambda\left(\vec{w}^{* T} \vec{w}\right)$.

By exchanging $i$ with $-i$, this relation becomes $\vec{w}^{T} \mathcal{V} \vec{w}^{*}=\lambda^{*}\left(\vec{w}^{T} \vec{w}^{*}\right)$; the sum of this relation and of the previous one gives $\lambda+\lambda^{*}=0$, because $\vec{w}^{* T} \vec{w}>0$

$$
\vec{w}^{* T} \mathcal{V} \vec{w}=w_{j}^{*} V_{j h} w_{h}=w_{h}^{*} V_{h j} w_{j}=w_{j} V_{h j} w_{h}^{*}=-w_{j} V_{j h} w_{h}^{*}=-\vec{w}^{T} \mathcal{V} \vec{w}^{*}
$$

where, in the second passage of the first equation, we have exchanged the names of the indexes $h$ and $j$. Obviously, from $\lambda+\lambda^{*}=0$ it follows that $\lambda$ has zero real part.

Proof of Property 2. The result $a=0$ has been proved in Property 1.

Let $\vec{w}=\vec{u}+i \vec{v}$; by multiplying $\mathcal{V} \vec{w}=i b \vec{w}$ on the left, times $\vec{w}^{T}$, we obtain $\vec{w}^{T} \mathcal{V} \vec{w}=i b\left(\vec{w}^{T} \vec{w}\right)$

but the left hand side of this relation is zero because $\mathcal{V}$ is skew-symmetric. It follows that $\vec{w}^{T} \vec{w}=0$, that is, $u^{2}-v^{2}+2 i \vec{u} \cdot \vec{v}=0$, from which our thesis follows.

Proof of Property 3. Let us prove this with the iterative procedure on the number $n$. It holds for $n=1$ (the unique skew-symmetric tensor is zero and 1 is its eigenvector corresponding to the eigenvalue zero). It holds also for $n=2$; in fact, if $\mathcal{V}=\left(\begin{array}{cc}0 & V^{12} \\ -V^{12} & 0\end{array}\right)$, with $V^{12} \neq 0$, the eigenvalues are $\pm i V^{12}$ and we have $\vec{u}_{1}=\left(\begin{array}{l}1 \\ 0\end{array}\right)$ and $\vec{v}_{1}=\left(\begin{array}{l}0 \\ 1\end{array}\right)$. On the other hand, if $V^{12}=0$, there is only the zero eigenvalue and we have $\vec{w}_{1}=\left(\begin{array}{l}1 \\ 0\end{array}\right)$ and $\vec{w}_{2}=\left(\begin{array}{c}0 \\ 1\end{array}\right)$.

Let us suppose now that the thesis holds for all the tensors of order less than $n$ and prove that it holds for tensors of order $n$.

If $\mathcal{V}$ has the zero eigenvalue, let $\vec{w}$ be an eigenvector corresponding to it; by eventually dividing it by its modulus, it becomes an unit vector. Let us complete it to an orthonormal basis $\vec{w}_{1}, \cdots, \vec{w}_{n-1}, \vec{w}$ and let $P$ be 
the matrix which has these vectors as columns. We have that $P^{T} \mathcal{V} P$ is skew-symmetric (It change sign when we pass to its transpose) and its last column is $P^{T} \mathcal{V} \vec{w}=\overrightarrow{0}$. In other words, the last column (and, consequently, also the last raw) of $P^{T} \mathcal{V} P$ is zero, that is, $P^{T} \mathcal{V} P=\left(\begin{array}{cc}V^{*} & \overrightarrow{0} \\ \overrightarrow{0}^{T} & 0\end{array}\right)$, with $V^{*}$ skew-symmetric and of order $n-1$. The Proposition holds for the tensor $V^{*}$, for the iterative hypothesis. Consequently, there is a basis for $\Re^{n-1}$ of orthonormal vectors $\vec{u}_{1}^{*}, \vec{v}_{1}^{*}, \cdots, \vec{u}_{r}^{*}, \vec{v}_{r}^{*}, \vec{w}_{1}^{*}, \cdots, \vec{w}_{n-1-2 r}^{*}$ such that $V^{*}\left(\vec{u}_{s}^{*}+i \vec{v}_{s}^{*}\right)=i b_{s}\left(\vec{u}_{s}^{*}+i \vec{v}_{s}^{*}\right)$ and $V^{*} \vec{w}_{p}^{*}=\overrightarrow{0}$. Let us consider now the vectors

$$
\vec{u}_{s}=P\left(\begin{array}{c}
\vec{u}_{s}^{*} \\
0
\end{array}\right), \quad \vec{v}_{s}=P\left(\begin{array}{c}
\vec{v}_{s}^{*} \\
0
\end{array}\right), \quad \vec{w}_{p}=P\left(\begin{array}{c}
\vec{w}_{p}^{*} \\
0
\end{array}\right)
$$

It follows that

$$
\begin{aligned}
& P^{T} \mathcal{V}\left(\vec{u}_{s}+i \vec{v}_{s}\right)=P^{T} \mathcal{V} P\left[\left(\begin{array}{c}
\vec{u}_{s}^{*} \\
0
\end{array}\right)+i\left(\begin{array}{c}
\vec{v}_{s}^{*} \\
0
\end{array}\right)\right]= \\
& =\left(\begin{array}{cc}
V^{*} & \overrightarrow{0} \\
\overrightarrow{0}^{T} & 0
\end{array}\right)\left[\left(\begin{array}{c}
\vec{u}_{s}^{*} \\
0
\end{array}\right)+i\left(\begin{array}{c}
\vec{v}_{s}^{*} \\
0
\end{array}\right)\right]= \\
& =i b_{s}\left(\begin{array}{c}
\vec{u}_{s}^{*}+i \vec{v}_{s}^{*} \\
0
\end{array}\right)=P^{T} i b_{s}\left(\vec{u}_{s}+i \vec{v}_{s}\right),
\end{aligned}
$$

from which $\mathcal{V}\left(\vec{u}_{s}+i \vec{v}_{s}\right)=i b_{s}\left(\vec{u}_{s}+i \vec{v}_{s}\right)$. Similarly,

$$
P^{T} \mathcal{V} \vec{w}_{p}=P^{T} \mathcal{V} P\left(\begin{array}{c}
\vec{w}_{p}^{*} \\
0
\end{array}\right)=\left(\begin{array}{cc}
V^{*} & \overrightarrow{0} \\
\overrightarrow{0}^{T} & 0
\end{array}\right)\left(\begin{array}{c}
\vec{w}_{p}^{*} \\
0
\end{array}\right)=\left(\begin{array}{c}
\overrightarrow{0} \\
0
\end{array}\right)
$$

from which $\mathcal{V} \vec{w}_{p}=\overrightarrow{0}$. Then $\vec{u}_{s}+i \vec{v}_{s}$ are eigenvectors of $\mathcal{V}$ and also $\vec{w}_{p}$. Let us prove now that $\vec{u}_{1}, \vec{v}_{1}, \cdots, \vec{u}_{r}, \vec{v}_{r}, \vec{w}_{1}, \cdots, \vec{w}_{n-1-2 r}, \vec{w}$ constitute an orthonormal basis of $\Re^{n}$. In fact, we have

$$
\begin{aligned}
& \vec{u}_{a} \cdot \vec{u}_{b}=\vec{u}_{a}^{T} \vec{u}_{b}=\left(\vec{u}_{a}^{* T} 0\right) P^{T} P\left(\begin{array}{c}
\vec{u}_{b}^{*} \\
0
\end{array}\right)=\left(\vec{u}_{a}^{* T} 0\right)\left(\begin{array}{c}
\vec{u}_{b}^{*} \\
0
\end{array}\right)= \\
& =\vec{u}_{a}^{*} \cdot \vec{u}_{b}^{*}=\delta_{a b}
\end{aligned}
$$

$\vec{v}_{a} \cdot \vec{v}_{b}=\delta_{a b}$, (it can be proved as the previous one, but with $\left(\vec{v}_{a}, \vec{v}_{b}\right)$ instead of $\left.\left(\vec{u}_{a}, \vec{u}_{b}\right)\right)$

$\vec{u}_{a} \cdot \vec{v}_{b}=0$, (it can be proved as the first relation, but with $\vec{v}_{b}$ instead of $\left.\vec{u}_{b}\right)$,

$\vec{u}_{a} \cdot \vec{w}_{p}=0$, (it can be proved as the first relation, but with $\vec{w}_{p}$ instead of 
$\left.\vec{u}_{b}\right)$,

$\vec{v}_{b} \cdot \vec{w}_{p}=0$, (it can be proved as the first relation, but with $\left(\vec{v}_{b}, \vec{w}_{p}\right)$ instead of $\left.\left(\vec{u}_{a}, \vec{u}_{b}\right)\right)$

$\vec{w}_{p} \cdot \vec{w}_{q}=\delta_{p q}$, (it can be proved as the first relation, but with $\left(\vec{w}_{p}, \vec{w}_{q}\right)$ instead of $\left.\left(\vec{u}_{a}, \vec{u}_{b}\right)\right)$

Finally, from the last column of $P^{T} P=I$ we have $P^{T} \vec{w}=\left(\begin{array}{l}\overrightarrow{0} \\ 1\end{array}\right)$ from which

$\vec{u}_{a} \cdot \vec{w}=\vec{u}_{a}^{T} \vec{w}=\left(\vec{u}_{a}^{* T} 0\right) P^{T} \vec{w}=\left(\vec{u}_{a}^{* T} 0\right)\left(\begin{array}{c}\overrightarrow{0} \\ 1\end{array}\right)=0$

$\vec{v}_{b} \cdot \vec{w}=0$, (it can be proved as the previous one, but with $\vec{v}_{b}$ instead of $\left.\vec{u}_{a}\right)$

$\vec{w}_{p} \cdot \vec{w}=0$ (it can be proved as the first one, but with $\vec{w}_{p}$ instead of $\vec{u}_{a}$ ). This completes the proof for this case.

The proof is similar when there is not the zero eigenvalue. In fact, let $\vec{u}_{1}+i \vec{v}_{1}$ be an eigenvector corresponding to the eigenvalue $i b$ with $b \neq 0$; for Property 2 we have that $\vec{u}_{1}$ and $\vec{v}_{1}$ have the same modulus; so, except for dividing them by their common modulus, they can be considered unit vectors. Moreover, also for Property 2, they are orthogonal. We complete them to an orthonormal basis of $\Re^{n}$ and call $P$ the matrix which has this basis as columns, taking $\vec{u}_{1}$ and $\vec{v}_{1}$ as first two columns. We have that $P^{T} \mathcal{V} P$ is skew-symmetric; moreover, the first two columns of $P^{T} \mathcal{V} P$ are $P^{T} \mathcal{V} \vec{u}_{1}=-b P^{T} \vec{v}_{1}=-b\left(\begin{array}{l}0 \\ 1 \\ \overrightarrow{0}\end{array}\right)=\left(\begin{array}{c}0 \\ -b \\ \overrightarrow{0}\end{array}\right) \quad$ and $P^{T} \mathcal{V} \vec{v}_{1}=b P^{T} \vec{u}_{1}=$ $b\left(\begin{array}{l}1 \\ 0 \\ \overrightarrow{0}\end{array}\right)=\left(\begin{array}{l}b \\ 0 \\ \overrightarrow{0}\end{array}\right)$,

respectively. (We have taken into account that $\mathcal{V}\left(\vec{u}_{1}+i \vec{v}_{1}\right)=i b\left(\vec{u}_{1}+i \vec{v}_{1}\right)$ is equivalent to

$\left\{\begin{array}{l}\mathcal{V} \vec{u}_{1}=-b \vec{v}_{1} \\ \mathcal{V} \vec{v}_{1}=b \vec{u}_{1}\end{array}\right.$ and the first two columns of $P^{T} P=I$ are $P^{T} \vec{u}_{1}=\left(\begin{array}{l}1 \\ 0 \\ \overrightarrow{0}\end{array}\right)$ and $P^{T} \vec{v}_{1}=\left(\begin{array}{c}0 \\ 1 \\ \overrightarrow{0}\end{array}\right)$. It follows that $P^{T} \mathcal{V} P=$ $\left(\begin{array}{ccc}0 & b & \overrightarrow{0}^{T} \\ -b & 0 & \overrightarrow{0}^{T} \\ \overrightarrow{0} & \overrightarrow{0} & V^{*}\end{array}\right)$, with $V^{*}$ skew-symmetric of order $n-2$. The Proposition holds for the tensor $V^{*}$, for the iterative hypothesis. Consequently, 
there is a basis for $\Re^{n-2}$ of orthonormal vectors $\vec{u}_{2}^{*}, \vec{v}_{2}^{*}, \cdots, \vec{u}_{r}^{*}, \vec{v}_{r}^{*}$ such that $V^{*}\left(\vec{u}_{s}^{*}+i \vec{v}_{s}^{*}\right)=i b_{s}\left(\vec{u}_{s}^{*}+i \vec{v}_{s}^{*}\right)$. Let us consider now the vectors

$$
\begin{aligned}
& \vec{u}_{s}=P\left(\begin{array}{c}
0 \\
0 \\
\vec{u}_{s}^{*}
\end{array}\right), \quad \vec{v}_{s}=P\left(\begin{array}{c}
0 \\
0 \\
\vec{v}_{s}^{*}
\end{array}\right) \text { and note that }
\end{aligned}
$$

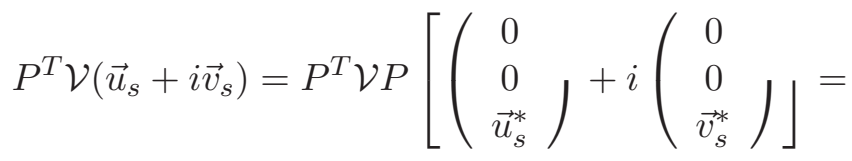

$$
\begin{aligned}
& \left(\begin{array}{ccc}
0 & b & \overrightarrow{0}^{T} \\
-b & 0 & \overrightarrow{0}^{T} \\
\overrightarrow{0} & \overrightarrow{0} & V^{*}
\end{array}\right)\left[\left(\begin{array}{c}
0 \\
0 \\
\vec{u}_{s}^{*}
\end{array}\right)+i\left(\begin{array}{c}
0 \\
0 \\
\vec{v}_{s}^{*}
\end{array}\right)\right]=
\end{aligned}
$$

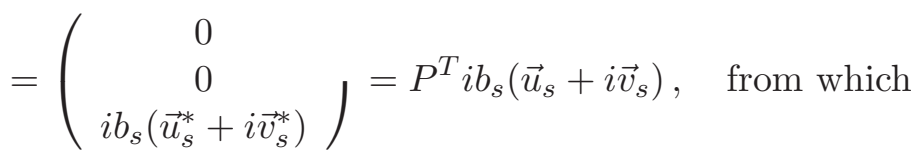

$\mathcal{V}\left(\vec{u}_{s}+i \vec{v}_{s}\right)=i b_{s}\left(\vec{u}_{s}+i \vec{v}_{s}\right)$ that is, $\vec{u}_{s}+i \vec{v}_{s}$ is an eigenvector.

Let us verify now that we have obtained an orthonormal basis. We have

$$
\vec{u}_{s} \cdot \vec{u}_{1}=\vec{u}_{s}^{T} \vec{u}_{1}=\left(\begin{array}{lll}
0 & 0 & \vec{u}_{s}^{* T}
\end{array}\right) P^{T} \vec{u}_{1}=\left(\begin{array}{lll}
0 & 0 & u_{s}^{* T}
\end{array}\right)\left(\begin{array}{c}
1 \\
0 \\
\overrightarrow{0}
\end{array}\right)=0,
$$

$\vec{v}_{s} \cdot \vec{u}_{1}=0$, (it can be proved as the previous one, but with $\vec{v}_{s}$ instead of $\vec{u}_{s}$,

$$
\vec{u}_{s} \cdot \vec{v}_{1}=\vec{u}_{s}^{T} \vec{v}_{1}=\left(\begin{array}{lll}
0 & 0 & \vec{u}_{s}^{* T}
\end{array}\right) P^{T} \vec{v}_{1}=\left(\begin{array}{lll}
0 & 0 & \vec{u}_{s}^{* T}
\end{array}\right)\left(\begin{array}{l}
0 \\
1 \\
\overrightarrow{0}
\end{array}\right)=0,
$$

$\vec{v}_{s} \cdot \vec{v}_{1}=0$, (it can be proved as the previous one, but with $\vec{v}_{s}$ instead of $\vec{u}_{s}$

$$
\begin{array}{r}
\vec{u}_{s} \cdot \vec{u}_{p}=\vec{u}_{s}^{T} \vec{u}_{p}=\left(\begin{array}{lll}
0 & 0 & \vec{u}_{s}^{* T}
\end{array}\right) P^{T} P\left(\begin{array}{c}
0 \\
0 \\
\vec{u}_{p}^{*}
\end{array}\right)=\left(\begin{array}{lll}
0 & 0 & u_{s}^{* T}
\end{array}\right)\left(\begin{array}{c}
0 \\
0 \\
\vec{u}_{p}^{*}
\end{array}\right)= \\
=\vec{u}_{s}^{*} \cdot \vec{u}_{p}^{*}=\delta_{s p},
\end{array}
$$

$\vec{u}_{s} \cdot \vec{v}_{p}=0$, (it can be proved as the previous one, but with $\vec{v}_{p}$ instead of $\left.\vec{u}_{p}\right)$,

$\vec{v}_{s} \cdot \vec{v}_{p}=\delta_{s p}$, (it can be proved as the first relation, but with $\left(\vec{v}_{s}, \vec{v}_{p}\right)$ instead of $\left.\left(\vec{u}_{s}, \vec{u}_{p}\right)\right)$.

This completes our proof. 
Proof of Property 4: Let $r_{i}$ be the number of linearly independent eigenvectors corresponding to the eigenvalue $\lambda_{i}$ and $\mu_{i}$ the multiplicity of this eigenvalue. It is well known that $r_{i} \leq \mu_{i}$ (In fact, for each fixed value of $i$, we may consider an invertible matrix $P$ (almost surely it is a complex matrix) which has the $r_{i}$ linearly independent eigenvectors corresponding to $\lambda_{i}$ as its first columns; we have that $P^{-1} \mathcal{V} P=$ $\left(\begin{array}{ccccc}\lambda_{i} & 0 & \cdots & 0 & \vec{v}_{1}^{T} \\ 0 & \lambda_{i} & \cdots & 0 & \vec{v}_{2}^{T} \\ \cdots & \cdots & \cdots & \cdots & \cdots \\ 0 & 0 & \cdots & \lambda_{i} & \vec{v}_{i}^{T} \\ \overrightarrow{0} & \overrightarrow{0} & \cdots & \overrightarrow{0} & V^{*}\end{array}\right)$ so that the characteristic equation for $\mathcal{V}$ is $0=|\mathcal{V}-\lambda \mathcal{I}|=\left|P^{-1}(\mathcal{V}-\lambda \mathcal{I}) P\right|=\left|P^{-1} \mathcal{V} P-\lambda \mathcal{I}\right|=\left(\lambda_{i}-\lambda\right)^{r_{i}}\left|V^{*}-\lambda \mathcal{I}\right|$. It follows that $\lambda_{i}$ has multiplicity $r_{i}$ if $\lambda_{i}$ is not a root of $\left|V^{*}-\lambda \mathcal{I}\right|=0$, otherwise its multiplicity is bigger than $r_{i}$.) Now we have that $\sum_{i} \mu_{i}=n$ and, for Property $3, \sum_{i} r_{i}=n$; it follows that $\sum_{i}\left(\mu_{i}-r_{i}\right)=n-n=0$ and consequently $\mu_{i}-r_{i} \geq 0$ implies $\mu_{i}-r_{i}=0$. This completes the proof of $\mu_{i}=r_{i}$.

Proof of Property 5: In fact, we have $\vec{w} \cdot \mathcal{V}(\vec{u}+i \vec{v})=0$ because $\vec{w}$ is an eigenvector of $\mathcal{V}$ corresponding to the eigenvalue $\lambda=0$. But we have also $\vec{w} \cdot \mathcal{V}(\vec{u}+i \vec{v})=\vec{w} \cdot i b(\vec{u}+i \vec{v})=b(i \vec{w} \cdot \vec{u}-\vec{w} \cdot \vec{v})$. Consequently, we have $\vec{w} \cdot \vec{u}=0$ and $\vec{w} \cdot \vec{v}=0 "$.

Proof of Property 6: Let $\vec{a}_{j}=\vec{u}_{j}+i \vec{v}_{j}$ and $\vec{a}_{2}^{*}=\vec{u}_{2}-i \vec{v}_{2}$ (that is, the complex conjugate of $\vec{a}_{2}$ ). We have

$\vec{a}_{1} \cdot \mathcal{V} \vec{a}_{2}=i b_{2} \vec{a}_{1} \cdot \vec{a}_{2}$ because $\vec{a}_{2}$ is an eigenvector of $\mathcal{V}$ corresponding to the eigenvalue $i b_{2}$.

But we have also that $\vec{a}_{1} \cdot \mathcal{V} \vec{a}_{2}=\vec{a}_{1}^{T} \mathcal{V} \vec{a}_{2}=\left(\vec{a}_{1}^{T} \mathcal{V} \vec{a}_{2}\right)^{T}=\vec{a}_{2}^{T} \mathcal{V}^{T} \vec{a}_{1}=$ $-\vec{a}_{2}^{T} \mathcal{V} \vec{a}_{1}=-\vec{a}_{2} \cdot \mathcal{V} \vec{a}_{1}=-i b_{1} \vec{a}_{2} \cdot \vec{a}_{1}$ where, in the last passage we have used the fact that $\vec{a}_{1}$ is an eigenvector of $\mathcal{V}$ corresponding to the eigenvalue $i b_{1}$, while in the second passage we have used the fact that a single number is a matrix of order 1 and this is equal to its transposed.

It follows that $\left(b_{1}+b_{2}\right) i\left(\vec{a}_{1} \cdot \vec{a}_{2}\right)=0$; but this must hold also with $\vec{a}_{2}^{*}$ instead of $\vec{a}_{2}$ (and, obviously, $-b_{2}$ instead of $b_{2}$ ), that is, $\left(b_{1}-b_{2}\right) i\left(\vec{a}_{1} \cdot \vec{a}_{2}^{*}\right)=0$. But the hypothesis $\left(b_{1}\right)^{2} \neq\left(b_{2}\right)^{2}$ implies $b_{1}+b_{2} \neq 0$ and $b_{1}-b_{2} \neq 0$. Consequently, our results become $\vec{a}_{1} \cdot \vec{a}_{2}=0, \vec{a}_{1} \cdot \vec{a}_{2}^{*}=0$. These equations can be written respectively as

$$
\left\{\begin{array}{l}
\vec{u}_{1} \cdot \vec{u}_{2}-\vec{v}_{1} \cdot \vec{v}_{2}=0 \\
\vec{u}_{1} \cdot \vec{v}_{2}+\vec{v}_{1} \cdot \vec{u}_{2}=0
\end{array} \quad, \quad\left\{\begin{array}{c}
\vec{u}_{1} \cdot \vec{u}_{2}+\vec{v}_{1} \cdot \vec{v}_{2}=0 \\
-\vec{u}_{1} \cdot \vec{v}_{2}+\vec{v}_{1} \cdot \vec{u}_{2}=0
\end{array} .\right.\right.
$$


Joining all these equations, we obtain $\vec{u}_{1} \cdot \vec{u}_{2}=0, \vec{u}_{1} \cdot \vec{v}_{2}=0, \vec{v}_{1} \cdot \vec{u}_{2}=0$ and $\vec{v}_{1} \cdot \vec{v}_{2}=0 "$.

Proof of Property 7: We can repeat the passage of the previous proof of Property 6 , but with $b_{1}=b_{2}=b$. It follows that eq. $(64)_{1}$ still holds, even if eq. $\left(64_{2}\right)$ is no more valid; this is because $b_{1}+b_{2}=2 b$, while $b_{1}-b_{2}=0$. In this way, property 7 is proved.

\section{References}

[1] G. F. Smith. On isotropic functions of symmetric tensor, skew symmetric tensor and vectors, Int. J. Engng Sci. 9 (1971), pp. 899-916. DOI: 10.1016/0020-7225(71)90023-1.

[2] C. C. Wang. On representations for isotropic functions, Arch. Ratl. Mech. Anal. 33 (1969), pp. 249-287. DOI: 10.1007/BF00281278 and 10.1007/BF00281279.

[3] J. P. Boeheler, On Irreducible Representations for Isotropic Scalar Functions, ZAMM 57 (1977), pp. 323-327. DOI: 10.1002/zamm.19770570608

[4] S.Pennisi, M.Trovato, On the Irreducibility of Professor G.F. Smith's Representations for isotropic functions, Int.J.Engng Sci. 25 (1987), pp.10591065. DOI: 10.1016/0020-7225(87)90097-8.

[5] S.Pennisi, On third order Tensor-valued Isotropic Functions, Int.J.Engng Sci. 30 (1992), pp. 679-692. DOI: 10.1016/0020-7225(92)90011-5.

[6] S. Pennisi, M. Trovato, Mathematical Characterization of functions underlying the principle of relativity, Le Matematiche, XLIV, (1989), pp. 173-204.

[7] S. Montisci, S. Pennisi, Representations Theorems for Scalar Functions in a 4-dimensional Euclidean Space, Int. J. of Pure and Appl. Math. 60 (2010), pp. 443-460.

[8] M. C. Carrisi, S. Montisci, S. Pennisi, Representations Theorems for Symmetric Tensorial Functions in a 4-dimensional Euclidean Space, IEJPAM 2 (2010), pp. 129-154.

[9] M. C. Carrisi, S. Montisci, S. Pennisi, Representations Theorems for SkewSymmetric Tensorial Functions in a 4-dimensional Euclidean Space, IEJPAM 2 (2010), pp. 155-181. 
[10] S. Montisci, S. Pennisi, Representations Theorems in a 4-dimensional Euclidean Space. A new case, IEJPAM 1 (2013), pp. 1-30. DOI: 10.12732/ijdea.v6i1.1

[11] M.C. Carrisi, S. Pennisi, T.Ruggeri, The Lagrangian view-point compared with the Eulerian one, in the framework of Extended Thermodynamics, Acta Applicandae Mathematicae,133, Issue 1 (2014), DOI: 10.1007/s10440-014-9921-0.

[12] M.C. Carrisi, S. Pennisi, Waves speeds in the macroscopic relativistic extended model with many moments, Meccanica, 49 (2014), pp. 1493-1506 DOI: $10.1007 / \mathrm{s} 11012-014-9933-\mathrm{x}$.

[13] Trovato M., Reggiani L., Quantum Maximum Entropy Principle for Fractional Exclusion Statistics, Phys. Rev. lett. 110 (2013), 020404-1-5 . DOI: 10.1103/PhysRevLett.110.020404.

[14] M.C. Carrisi, S. Pennisi, Extended Thermodynamics of Charged Gases with Many Moments: An Alternative Closure, J. Math. Phys., 54 (9) (2013), art. no. 09301 , DOI: 10.1063/1.4821086

[15] M.C. Carrisi, S. Montisci, S. Pennisi, Entropy Principle and Galilean Relativity for Dense Gases, the General Solution without Approximations, Entropy 15 (3) (2013), pp. 1035-1056. DOI: 10.3390/e15031035.

[16] M.C. Carrisi, S. Pennisi, Extended Thermodynamics of charged gases with many moments, J. Math. Phys. 54, (2) (2013), art. no. 023101 DOI: $10.1063 / 1.4789544$.

[17] M. Trovato, L. Reggiani, Maximum entropy principle and hydrodynamic models in statistical mechanics. Riv. Nuovo Cimento Soc. Ital. Fis. 35(2012), pp. 99 - 266

[18] M.C. Carrisi. A generalized kinetic approach for the study of relativistic electron beams. Acta Applicandae Mathematicae 122 (2012), 107-116, DOI: $10.1007 / \mathrm{s} 10440-012-9730-2$.

[19] M.C. Carrisi, S. Pennisi, Some open problems in non-linear extended thermodynamics and their possible solutions. Ricerche di Matematica 60 (2012), 45-56, DOI: 10.1007/s11587-010-0095-4.

[20] Trovato M., Reggiani L., Quantum maximum-entropy principle for closed quantum hydrodynamic transport within a Wigner function formalism, Phys. Rev. E 84 (2011), 061147- 1-29 DOI: 10.1103/PhysRevE.84.061147. 
[21] Trovato M., Reggiani L., Quantum maximum entropy principle for a system of identical particles, Phys. Rev. E 81 (2010), 021119-1-11 DOI: 10.1103/PhysRevE.81.021119.

[22] Marras, M., Vernier Piro, S., Explicit estimates for blow-up solutions to parabolic systems under nonlocal boundary conditions, Comptes Rendus de L'Academie Bulgare des Sciences 67 (4), (2014), pp. 459-466

[23] Marras, M., Porru, G., Vernier-Piro, S., Optimization problems for eigenvalues of p-Laplace equations, Journal of Mathematical Analysis and Applications 398 (2), (2013), pp. 766-775

[24] Gramchev, T., Marras, M., Vernier-Piro, S., Extinction Phenomena for Parabolic Systems, Springer Proceedings in Mathematics and Statistics $\mathbf{4 7}$ (2013), pp. 561-570 DOI: 10.1007/978-1-4614-7333-6-51.

[25] Marras, M., Piro, S.V., Blow-up phenomena in reaction-diffusion systems, Discrete and Continuous Dynamical Systems- Series A 32 (11) (2012), pp. 4001-4014,

[26] Marras, M., Porru, G., Estimates and uniqueness for boundary blow-up solutions of P-laplace equations, Electronic Journal of Differential Equations 2011, pp. $1-10$ (2011)

[27] Marras, M., Vernier Piro, S., Bounds for blow-up time in nonlinear parabolic systems, Discrete and Continuous Dynamical Systems- Series A (SUPPL.) (2011), pp. 1025-1031

[28] T.Ruggeri, M. Trovato, Hyperbolicity in extended thermodynamics of Fermi and Bose gases, Continuum Mech. Thermodyn. 16 (2004), 551-576 DOI: 10.1007/s00161-004-0180-6.

[29] S. Lombardo, G. Mulone, M. Trovato, Nonlinear stability in reactiondiffusion systems via optimal Lyapunov functions, Journal Of Mathematical Analysis And Applications 342 (2008), 461-476 DOI: 10.1016/j.jmaa.2007.12.024.

[30] M. Trovato, P.Falsaperla, L. Reggiani, Maximum entropy principle for nonparabolic hydrodynamic transport in semiconductor devices, Journal Of Applied Physics 86 (1999), 5906-5908 DOI: 10.1063/1.371610. 
